\title{
Radio, Hard X-Ray, and Gamma-Ray Emissions Associated with a Far-Side Solar Event
}

\author{
V. Grechnev ${ }^{1}$ (D) V. Kiselev ${ }^{1}$. \\ K. Kashapova ${ }^{1,2}$ (D) A. Kochanov ${ }^{1,2}$. \\ I. Zimovets ${ }^{3,4,5}$. A. Uralov ${ }^{1}$ B. Nizamov Nit7 $^{6,7}$. \\ I. Grigorieva ${ }^{8}$ - D. Golovin ${ }^{3}$ - M. Litvak L $^{3}$. \\ I. Mitrofanov ${ }^{3}$ - A. Sanin ${ }^{3}$ \\ Received ; accepted

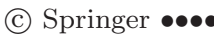

\begin{abstract}
The SOL2014-09-01 far-side solar eruptive event produced hard electromagnetic and radio emissions observed with detectors at near-Earth vantage points. Especially challenging was a long-duration $>100 \mathrm{MeV} \gamma$-ray burst probably produced by accelerated protons exceeding $300 \mathrm{MeV}$. This observation raised a question of how high-energy protons could reach the Earth-facing solar surface. Some preceding studies discussed a scenario in which protons accelerated by a CME-driven shock high in the corona return to the solar surface. We continue with the analysis of this challenging event, involving radio images from the Nançay Radioheliograph and hard X-ray data from the High Energy Neutron Detector (HEND) of the Gamma-Ray Spectrometer onboard the Mars Odyssey space observatory located near Mars. HEND recorded unocculted flare emission. The results indicate that the emissions observed from the Earth's direction
\end{abstract}

\footnotetext{
1 Institute of Solar-Terrestrial Physics SB RAS, Lermontov St. 126A, Irkutsk 664033, Russia email: grechnev@iszf.irk.ru ${ }^{2}$ Irkutsk State University, Gagarin Blvd. 20, Irkutsk 664003, Russia

3 Space Research Institute RAS, Profsoyuznaya St. 84/32, Moscow 117997, Russia email: ivanzim@iki.rssi.ru

4 State Key Laboratory of Space Weather, National Space Science Center (NSSC) of the Chinese Academy of Sciences, No. 1 Nanertiao, Zhongguancun, Haidian District, Beijing, 100190, China

5 International Space Science Institute - Beijing (ISSI-BJ), No. 1 Nanertiao, Zhongguancun, Haidian District, Beijing, 100190, China

6 Faculty of Physics, Lomonosov Moscow State University, Moscow, 119992 Russia

7 Sternberg Astronomical Institute, Lomonosov Moscow State University, Universitetskii Pr. 13, Moscow, 119992 Russia

8 Main Astronomical (Pulkovo) Observatory RAS, Pulkovskoe Sh. 65, St. Petersburg 196140, Russia email: irina.2014.irina@mail.ru
} 
were generated by flare-accelerated electrons and protons trapped in static long coronal loops. Their reacceleration is possible in these loops by a shock wave, which was excited by the eruption, being initially not CME-driven. The results highlight the ways to address remaining questions.

Keywords: Flares, Energetic Particles; Magnetic Fields, Corona; Radio Bursts; Waves, Shock; X-Ray Bursts

\section{Introduction}

The source of solar energetic particles (SEPs) produced in solar eruptive-flare events is a subject of long-standing debate. SEPs consist of different species dominated by protons. Two sources of accelerated protons have been considered (see, e.g., Kahler, 2001; Kallenrode, 2003; Aschwanden, 2012; Reames, 2013). One presumable origin of accelerated protons is associated with flare processes in solar active regions manifested in X-rays and microwaves. Another source is related to a bow-shock driven by the outer surface of a super-Alfvénic coronal mass ejection (CME). Many indications have been considered to identify the elusive source of accelerated protons. One of them is $\gamma$-ray emission, which was mostly observed concurrently with other flare emissions and seemingly favored the acceleration of protons in flares along with electrons (e.g. Ramaty and Mandzhavidze, 2000; Livshits and Belov, 2004; Chupp and Ryan, 2009; Kurt et al., 2010; Vilmer, MacKinnon, and Hurford, 2011).

Flare emissions are observed in a wide electromagnetic range, from radio waves up to high-energy $\gamma$-rays. Gyrosynchrotron emission observed in the radio range and a broadband hard X-ray (HXR) and $\gamma$-ray bremsstrahlung continuum are produced by accelerated electrons. Accelerated protons and heavier ions can be recognized from discrete $\gamma$-ray lines. Of special interest is the $\pi^{0}$-decay emission. Neutral pions appear in proton-proton collisions, when the proton energy exceeds $300 \mathrm{MeV}$, and they rapidly decay into two photons, producing a Dopplerbroadened wide enhancement in the $\gamma$-ray spectrum around $100 \mathrm{MeV}$ on top of the bremsstrahlung continuum (e.g. Ramaty, Kozlovsky, and Lingenfelter, 1975; Hudson and Ryan, 1995; Vilmer, MacKinnon, and Hurford, 2011). Thus, the $\pi^{0}$-decay emission is a direct indication of protons accelerated to high energies. Observations and identification of this $\gamma$-ray emission are only possible with a high sensitivity and sufficient spectral measurements at high energies. For this reason, the total number of events with a confident identification of the $\pi^{0}$-decay emission was fewer than 20 in the past (e.g. Ryan, 2000; Grechnev et al., 2008b; Chupp and Ryan, 2009; Kurt et al., 2010; Kuznetsov et al., 2011).

Being temporally close to flare emissions produced by accelerated electrons, discrete nuclear $\gamma$-ray lines, and especially the $\pi^{0}$-decay emission, have been considered as evidence of proton acceleration in flares. On the other hand, $\gamma$-ray emission much longer than the HXR burst was observed in a few events (e.g. Forrest et al., 1985; Akimov et al., 1996; Ryan, 2000). A challenge to the flarerelated origin of $\gamma$-ray emission was provided by the observation of $\gamma$-ray emission from an event behind the solar limb. To explain this phenomenon, Cliver, Kahler, 
and Vestrand (1993) proposed that protons accelerated by a CME-driven shock wave on an open magnetic field partly escaped into interplanetary space and partly returned to the solar surface, precipitating far from the flare region.

With the advent of the Fermi Gamma-Ray Space Telescope mission in 2008, high-sensitivity $\gamma$-ray observations become available with a comprehensive spectral information and coordinate measurements of $\gamma$-ray photons at $>100 \mathrm{MeV}$ by the Large Area Telescope (LAT: Atwood et al., 2009). Although it is a non-solar mission, Fermi also provides rich information for solar studies. Fermi has shown that $\gamma$-ray emissions are quite common in solar flares. Thirty long-duration $\gamma$ ray events have been observed (Share et al., 2017). Pesce-Rollins et al. (2015) reported on the detection by Fermi/LAT of high-energy $\gamma$-ray emissions from three behind-the-limb solar flares on 11 October 2013, 6 January 2014, and 1 September 2014. These events were addressed by Ackermann et al. (2017). The $\pi^{0}$-decay emission was identified with confidence in two of them, SOL2013-1011 and SOL2014-09-01. The authors invoked the idea of Cliver, Kahler, and Vestrand (1993) to interpret these emissions.

Plotnikov, Rouillard, and Share (2017) elaborated on this idea in their analysis of the three events. Among the issues analyzed, by means of three-dimensional reconstructions of coronal shock fronts, the authors showed the events' magnetic connectivity to the Earth-facing solar surface. Ackermann et al. (2017) and Plotnikov, Rouillard, and Share (2017) considered coronal shocks to be driven by fast CMEs and emphasized that the CME and associated shock wave were fastest in the 1 September 2014 event, where the high-energy $\gamma$-ray emission was strongest.

On the other hand, Hudson (2017) pointed out basic problems of the scenario proposed by Cliver, Kahler, and Vestrand (1993). First, a large mirror ratio at the base of an open coronal structure prevents the back-precipitation of particles from large coronal heights, so that only a small part of the protons is able to return to the Sun in this scheme. Second, the total number of high-energy protons estimated for a set of SEP events appears to be insufficient to sustain the high-energy $\gamma$-rays in the events addressed by Ackermann et al. (2017).

To explain the long-duration $\gamma$-rays from occulted events, Hudson (2017) considered two options. In the "Lasso" scenario, some SEPs are captured in a noose, which extends to several solar radii and then retracts. In this scenario, trapped particles acquire energy due to the betatron acceleration and first-order Fermi process. The second option that he proposed is a "coronal thick target" scenario, in which protons trapped in a static volume generate pions and $\gamma$-ray continuum. As Hudson (2017) estimated, this can proceed for a few hours.

Analyzing the dynamic evolution of the global magnetic field and the shock wave considered to be CME-driven, Jin et al. (2018) simulated the CME in the 1 September 2014 event by using a global MHD model. The authors concluded that particles responsible for the high-energy $\gamma$-ray emission were accelerated in the CME environment and escaped the shock downstream region along magnetic fields connected to the solar surface far away from the flaring region.

So one has to conclude that, in spite of the rich observational data available at present and a lot of efforts applied, the source of accelerated protons escapes 
identification. Furthermore, examining the "flare vs. CME-driven shock" alternative, the researchers base their considerations on a simplified traditional scheme of the bow-shock excitation by the outer surface of a fast CME. However, recent studies show that coronal shock waves are initially excited by sharply erupting flux-ropes inside the developing CMEs, while reconnection processes underneath produce a flare (see, e.g., Grechnev et al., 2016, 2018 for details and review). The flare, CME, and shock-formation processes turn out to be tightly associated, which determines a close relation between the characteristics of flares, CMEs, and shock waves. The situation gets still more complicated.

If flare processes are actually responsible for acceleration of protons, then parameters of CMEs are misleading. If shock-acceleration is at work, then the acceleration starts earlier and at lower altitudes than assumed previously. If both sources are implicated, then untangling their contributions is still more difficult.

Keeping in mind these circumstances, we continue with the analysis of the 1 September 2014 event. It was observed from different vantage points. From the Earth's direction, the HXR burst was observed by the Fermi Gamma-ray Burst Monitor (GBM: Meegan et al., 2009) and the Wind/Konus GammaRay Burst Experiment (Aptekar et al., 1995). A radio burst dominated by the gyrosynchrotron (GS) emission at frequencies $>300 \mathrm{MHz}$ was recorded by the Radio Solar Telescope Network (RSTN: Guidice, 1979; Guidice et al., 1981), while its source was observed at the Nançay Radioheliograph (NRH: Kerdraon and Delouis, 1997). The GS burst was considered by Ackermann et al. (2017) and Carley et al. (2017). The unocculted flare emission was recorded from the Martian direction by the High Energy Neutron Detector (HEND) of the GammaRay Spectrometer onboard the Mars Odyssey space observatory (Boynton et al., 2004). The SOL2014-09-01 event was not listed in the HEND catalog (Livshits et al., 2017); nevertheless, HEND actually observed it.

Based on these data, we analyze the electromagnetic emissions observed, endeavor to figure out their possible sources, try to understand the causes of the long-lasting emissions, and reveal the history and possible role of the shock wave. In this way, we pursue understanding which scenarios of those proposed match the observations, specifying and refining some of the results and conclusions drawn previously.

Section 2 addresses electromagnetic emissions observed in the event and their probable sources. Section 3 analyzes shock waves and their kinematics. Section 4 discusses the results and their interpretation. Section 5 summarizes the findings and presents the conclusion.

\section{Electromagnetic Emissions and Their Sources}

\subsection{Overview of the Event}

The eruptive flare occurred in an active region (AR) located behind the east limb at a position of N14E126 estimated by Ackermann et al. (2017) or N14 E129 according to our estimate. The AR was numbered 12158 when it became visible from Earth. The flare was visible from different vantage points. It was observed 

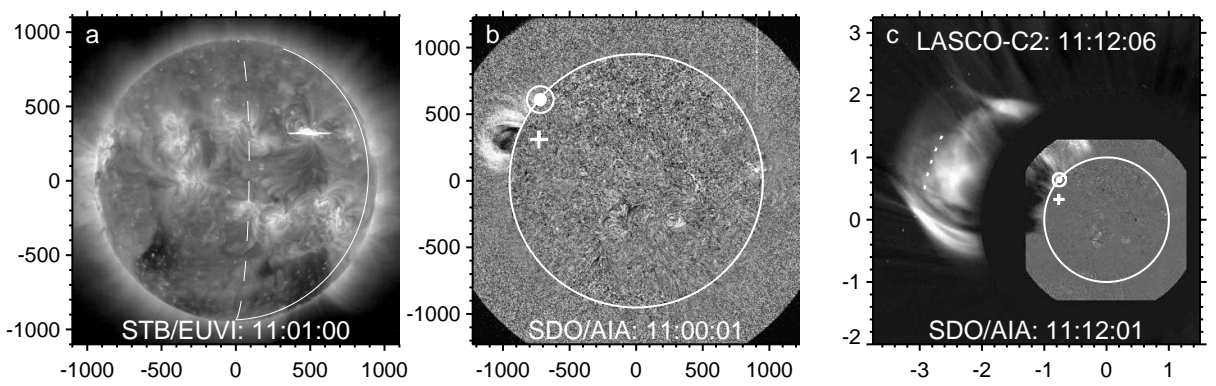

Figure 1. The SOL2014-09-01 event observed from different vantage points. a) The flare observed by STEREO-B/EUVI in $195 \AA$. The meridian outlined with solid arc is at a heliolongitude of $-90^{\circ}$ approximately corresponding to the east limb visible from Earth. The dashed arc at $-155.3^{\circ}$ corresponds to the east limb visible from Mars. b) The early CME lift-off in an SDO/AIA $211 \AA$ image ratio. The cross denotes the projected position of the flare site. c) $\mathrm{CME}$ in a LASCO-C2 image with an inserted co-temporal AIA $211 \AA$ image ratio. The dotted arc outlines the $\mathrm{CME}$ core at position angles $73^{\circ} \pm 10^{\circ}$ according to the measurements presented in Figure 2f: The dot in panels b and c denotes the Fermi/LAT > $100 \mathrm{MeV}$ emission centroid position with the $68 \%$ error circle (from Ackermann et al., 2017). The axes indicate the distance from solar disk center in arcseconds in panels a and b and in solar radii in panel c.

from the STEREO-B spacecraft of the twin Solar Terrestrial Relations Observatory (STEREO: Kaiser et al., 2008). STEREO-B was located $161^{\circ}$ eastward from Earth. The vantage point of HEND onboard the Mars Odyssey was located $65.3^{\circ}$ eastward from Earth.

Figure 17 presents the flare (bright streak) as observed by $195 \AA$ by the $E x$ treme Ultraviolet Imager (EUVI: Howard et al., 2008) onboard STEREO-B. As the figure shows, the flare emission was unocculted for STEREO-B and HEND. According to Plotnikov, Rouillard, and Share (2017), the flare started in soft Xrays at about 10:54 and peaked at about 11:11 (all times henceforth are adjusted to observations from $1 \mathrm{AU}$ and referred to UTC). The GOES importance of the flare estimated indirectly from STEREO-B/EUVI $195 \AA$ data ranged from X1.0 (Chertok, Belov, and Grechnev, 2015) to X2.4 estimated by Ackermann et al. (2017) using the method of Nitta et al. (2013).

Figure 1b exemplifies the observations by the Atmospheric Imaging Assembly (AIA: Lemen et al., 2012) onboard the Solar Dynamic Observatory (SDO) located at a near-Earth vantage point. For the analysis we mostly use the quarterresolution level 1.5 synoptic AIA data available at jsoc.stanford.edu/data/aia/synoptic/ in steps of two minutes. The AIA $211 \AA$ image ratio in Figure 10 presents the early lift-off of the CME. The projected position of the far-side active region is denoted by the cross. The dot denotes the Fermi/LAT $>100 \mathrm{MeV}$ emission centroid position with the $68 \%$ error circle measured by Ackermann et al. (2017). The error circle characterizes the measurement accuracy and should not be confused with the scatter in the positions of individual $\gamma$-ray photons, which occupy a very large area of several solar disks. The Fermi/LAT centroid position is commented on in Section 4.2

Figure 1, shows the CME observed by the Large Angle Spectroscopic Coronagraph (LASCO: Brueckner et al., 1995) onboard the Solar and Heliospheric 
Observatory (SOHO) with an inserted co-temporal $211 \AA$ image ratio. The dotted arc outlines the CME core at position angles $73^{\circ} \pm 10^{\circ}$ according to the measurements presented in Figure 2f. The average speed of a fastest CME feature measured in the online CME catalog (cdaw.gsfc.nasa.gov/CME_list/: Yashiro et al., 2004) at position angles from $76^{\circ}$ to $60^{\circ}$ was about $1900 \mathrm{~km} \mathrm{~s}^{-1}$ with a strong average deceleration of $-240 \mathrm{~ms}^{-2}$. These properties indicate that the measurements in the CME catalog are related to a shock wave (Grechnev et al., 2011a).

It is difficult to detect any erupting feature in EUVI $195 \AA$ images, whereas rare imaging in different EUVI channels missed the event. Nevertheless, the AIA $131 \AA$ images in Figures $2 \mathrm{a}-2$ reveal a blob rising radially from behind the limb. The dashed lines bound the angular extend of the blob $73^{\circ} \pm 5^{\circ}$ with a central position angle denoted by the straight black line. After an apparent fast initial three-dimensional expansion, the blob did not exceed laterally the dashed lines by 11:02:00.

The blob is only visible in $131 \AA$ and not detectable in any other channels. The temperature sensitivity characteristics of the AIA channels (Lemen et al., 2012) thus suggest a blob temperature of about $10 \mathrm{MK}$. Most likely, this was an erupting flux rope. Hot flux ropes have previously been observed in $131 \AA$ (e.g. Cheng et al., 2011; Zimovets et al., 2012; Patsourakos, Vourlidas, and Stenborg, 2013; Grechnev et al., 2016). The structure of the blob is indiscernible; nevertheless, the AIA observations allow us to infer its kinematics.

Figures $2 \mathrm{r}-2 \mathrm{~h}$ present probable kinematical plots of the blob inferred from the AIA $131 \AA$ observations within the shaded interval by fitting an analytic function to the observed motion. We used a Gaussian acceleration pulse, while its actual shape is uncertain because of the double integration in the transition from the acceleration to the distance-time dependence. The technique to infer the kinematics is similar to that used by Grechnev et al. (2015, 2016, 2018).

The initial velocity of the blob was close to zero. Its final velocity is determined by the position of the CME core in the first LASCO-C2 image, where it appeared; the CME frontal structure behind the wave trace corresponds to the pre-eruption arcade enveloping the flux-rope progenitor. The difference between the final and initial velocities is equal to the integral over the acceleration pulse. Its duration (and maximum) is adjusted in attempts to reproduce, on average, the accelerating motion of the blob barely visible within the AIA field of view.

The fit is shown in Figures 2 - 2 by the black arcs. The blob underwent a maximum acceleration around 10:59:40 and reached a final speed of $1900 \pm$ $150 \mathrm{kms}^{-1}$. The uncertainty in the duration and maximum of the Gaussian acceleration pulse is within a factor of two.

Figure 2 shows that when the acceleration ceased, the blob lagged behind the CME leading edge measured in the CME catalog by a factor of 1.45 at the first CME appearance in the LASCO-C2 field of view. The leading edge of the blob at that time according to the kinematics presented in Figures $2 \mathrm{~F}-2 \mathrm{~h}$ is denoted in Figure 1; with a dotted arc, which corresponds to the CME core. We stated the association of an erupting flux rope with the CME core previously that does not contradict the traditional idea relating the flux rope to the cavity; the flux-rope forms in the cavity from the structures of the core in the course of a time-extended process (Kuzmenko and Grechnev, 2017). 

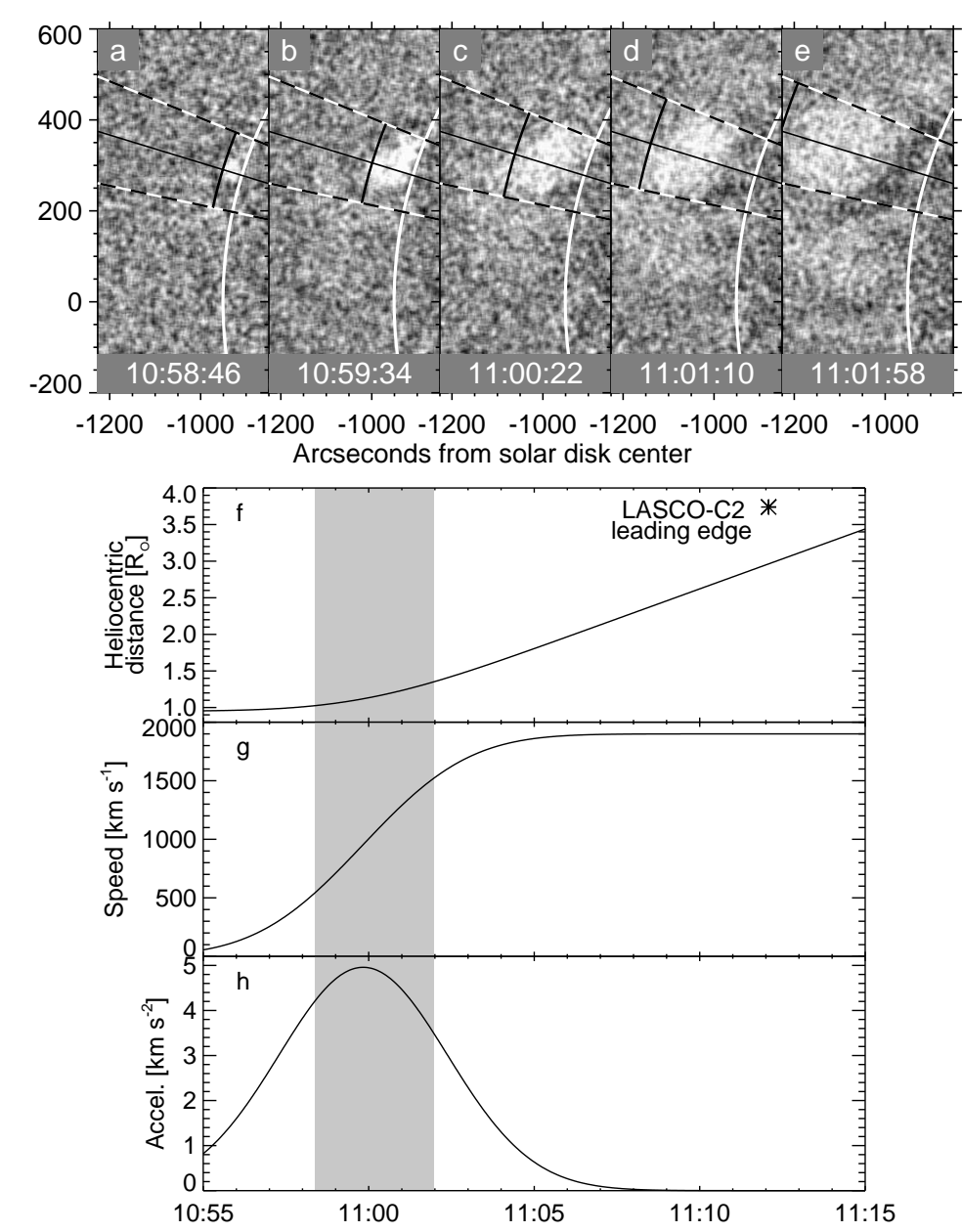

Figure 2. a-e) Rising blob in AIA $131 \AA$ image ratios (each divided by a fixed pre-event image observed at 10:58:22). The white circles denote the solar limb. The black arcs outline the leading edge of the blob. The dashed lines bound the angular extent of the blob $73^{\circ} \pm 5^{\circ}$ with a central position angle denoted by the straight black line. $\mathrm{f}-\mathrm{h}$ ) Probable kinematical plots of the blob. The asterisk in panel $\mathrm{f}$ represents the first CME measurement in the CME catalog. The shading represents the interval where the blob was measured within the AIA field of view.

\subsection{Temporal Profiles of the Bursts}

Figure 3 presents the bursts observed in microwaves, HXR, and $>100 \mathrm{MeV} \gamma$ rays from different vantage points. Figure 3 a shows the unocculted HXR burst recorded by HEND in a range of $50-800 \mathrm{keV}$ with a temporal sampling of $20 \mathrm{~s}$. The HXR burst comprised two overlapping impulsive peaks, each of about 1.5 minutes, followed by a long-lasting weaker gradual decay. The first peak occurred around 11:02:20 and the second around 11:04:30.

The microwave burst observed by RSTN from Earth exhibits the first minor peak corresponding to the first unocculted HXR peak around 11:02:20. The 


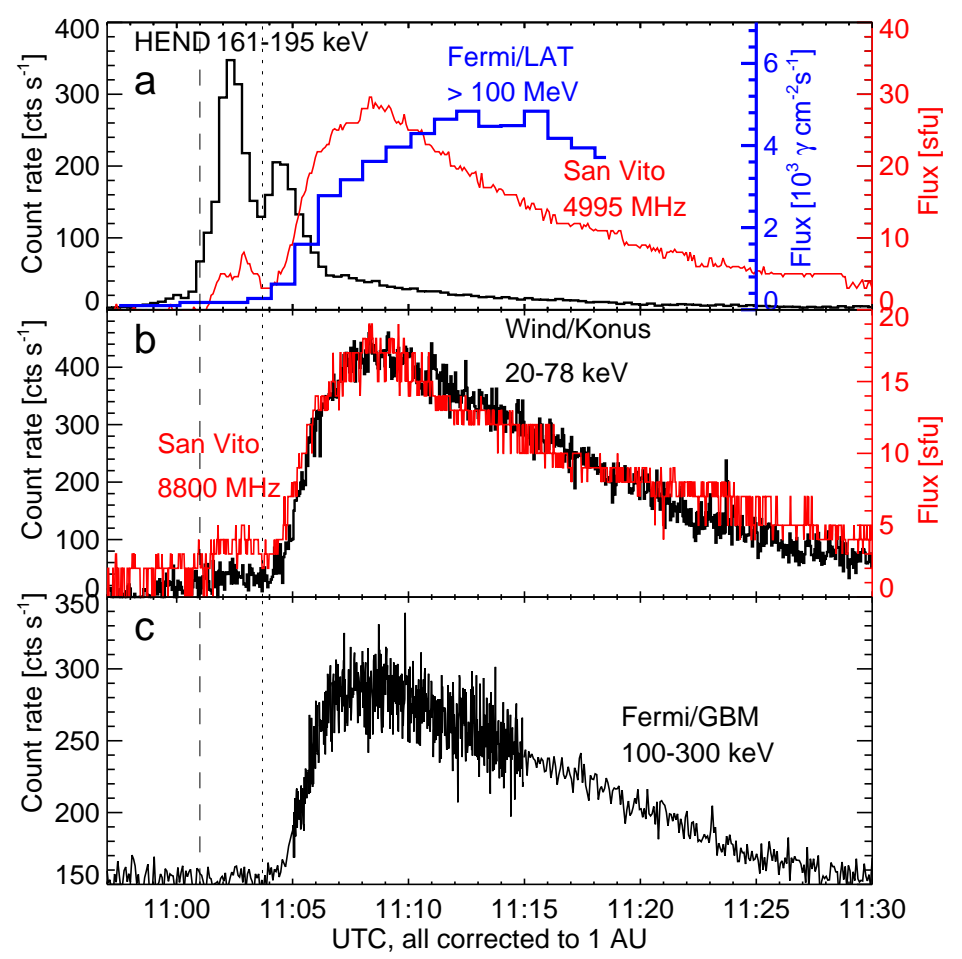

Figure 3. Electromagnetic emissions observed from different vantage points. a) Unocculted-flare HXR burst recorded from the Martian direction by HEND (black), microwave burst at $5 \mathrm{GHz}$ recorded in San Vito (red), and $>100 \mathrm{MeV} \gamma$-ray burst recorded by Fermi/LAT (thick-blue). b) Comparison of the HXR (Wind/Konus, black) and microwave (San Vito 8.8 GHz, red) bursts observed from the Earth's direction (similar to Figure 5 in Ackermann et al., 2017). c) Higher-energy HXR burst recorded by Fermi/GBM (similar to Figure 3 in Plotnikov, Rouillard, and Share, 2017).

main microwave burst started nearly simultaneously with the second unocculted HXR peak around 11:04:30 but looks strongly "stretched" and lasted about half an hour. This behavior suggests confinement of emitting electrons in a magnetic trap after an initial impulsive injection during the second unocculted HXR peak. The behavior of the high-energy $\gamma$-ray burst appears to be similar to the microwave burst; it started nearly simultaneously with the second HXR peak, being "stretched" still more strongly.

The microwave burst and a lower-energy HXR burst observed from the Earth's direction by Wind/Konus (Figure 3b) were almost identical in shape, with the first minor peak and main long-duration burst. The main HXR burst observed by Fermi/GBM at higher energies was similar, while the first minor peak was indistinct.

The photon spectrum index $[\gamma]$ estimated from the HEND data was 3.27 for the first peak and 3.13 for the second peak and then gradually hardened down to $\approx 2.2$ at $11: 15$, resembling the "soft-hard-harder" spectral behavior (Kiplinger, 1995). The photon-index error caused by the dead-time correction uncertainty does not exceed 0.3. Thus, the spectrum indices of the two unocculted HXR 


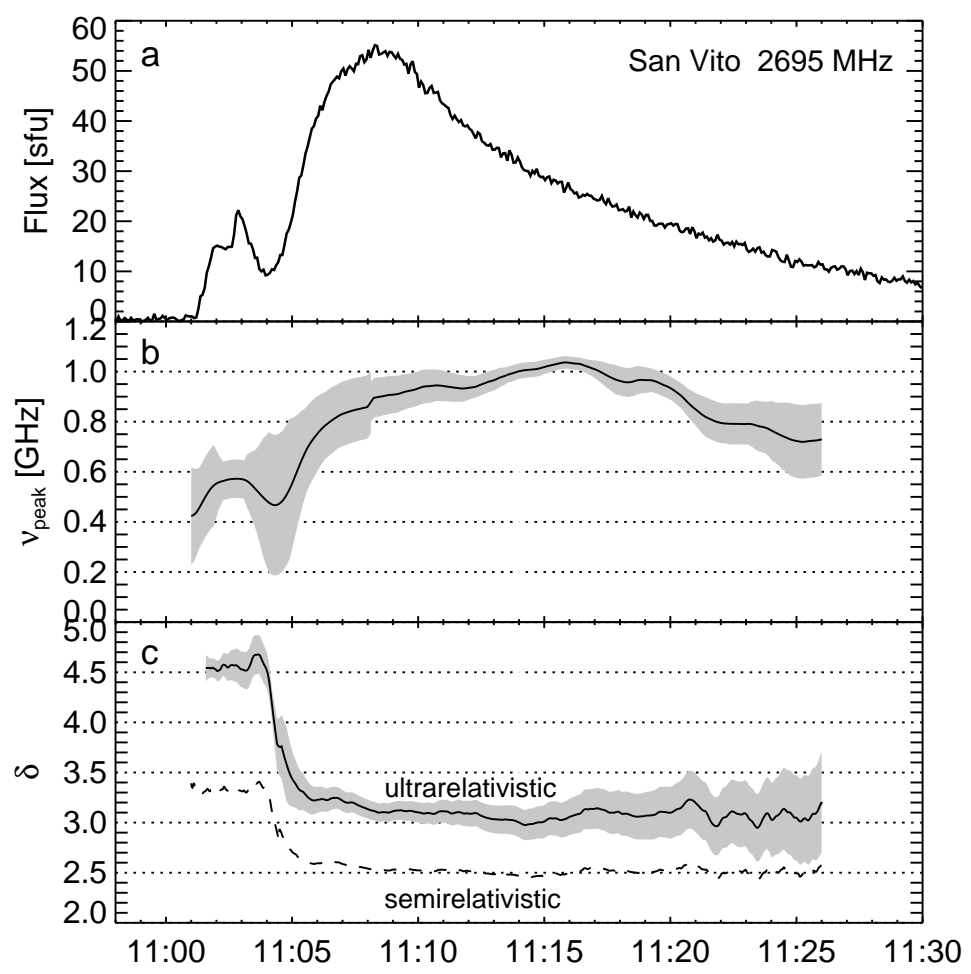

Figure 4. Evolution of the microwave emission during the event. a) Total-flux temporal profile recorded in San Vito at $2.7 \mathrm{GHz}$. b) Variations of the microwave peak frequency. c) Power-law index of microwave-emitting electrons estimated from the slope of the GS spectrum (ultrarelativistic limit solid, semirelativistic case dashed). The error ranges are shown with gray shadings.

peaks were almost identical. On the other hand, the main long-duration burst visible from the Earth's direction was obviously harder in HXR than the main burst. According to Ackermann et al. (2017), the emission spectrum integrated between 11:02 and 11:20 corresponded to a single power-law from $30 \mathrm{keV}$ to about $10 \mathrm{MeV}$ with an index of 2.06. This value is close to the index estimated from HEND data for a later stage of the event.

Figure 4 presents microwave spectral characteristics in comparison with the temporal profile at $2695 \mathrm{MHz}$. The variations in the peak frequency $\left[\nu_{\text {peak }}\right]$ of the GS emission shown in Figure 4b were computed by fitting in the $\log -\log$ scale of a parabola to an instantaneous set of samples recorded at different frequencies in San Vito (see, e.g., White et al., 2003; Grechnev et al., 2013a). The shading represents the measurement errors caused by the background-level uncertainties and noise and does not include the calibration uncertainties, which are not known. The peak frequency in the first minor peak was about $500 \mathrm{MHz}$. During the main burst, $\nu_{\text {peak }}$ increased but did not show large variations, being within $700-1000 \mathrm{MHz}$.

Figure 4 presents the power-law index of the electron energy density (electron number) spectrum estimated from the microwave index $[\alpha]$ at optically 
thin frequencies considerably exceeding $\nu_{\text {peak }}$. Usually such estimates invoke the semirelativistic approximation by Dulk and Marsh (1982) (gyrosynchrotron emission), $\delta=1.36-1.1 \alpha$, where $\alpha$ is signed and $\delta$ is always positive. The dashed line in Figure 4 4 represents $\delta$ estimated in this way. On the other hand, according to Ackermann et al. (2017) and Plotnikov, Rouillard, and Share (2017), a single-power-law electron spectrum exceeded $10 \mathrm{MeV}$ during the main burst and extended up to about $7 \mathrm{MeV}$ during the first minor peak (Carley et al., 2017). Thus, the estimate for the ultrarelativistic limit (synchrotron emission) might be more applicable. In this case, $\delta=1-2 \alpha$ (Dulk, 1985). Figure 4 shows this estimate by the solid line with uncertainties represented by the gray shading. While the synchrotron emission matches the situation better, we use the "gyrosynchrotron" term following the tradition.

For the thick-target emission in the non-relativistic limit corresponding to the HEND observations, the electron number index $\delta=\gamma+1.5 \approx 4.7(+0.5$ relative to the electron-flux spectrum; see Silva, Wang, and Gary, 2000; White et al., 2011). This value is close to the power-law index of microwave-emitting electrons in the first minor peak (the GS emission is mainly produced by the highenergy part of the electron spectrum). However, much harder electron spectrum is suggested by the main burst. Progressive hardening of the electron spectrum down to $\delta-3 / 2$ is possible in a magnetic trap, where particles are injected continuously (Melrose and Brown, 1976; Melnikov and Magun, 1998; Metcalf and Alexander, 1999). However, if we are really dealing here with trapping, then the main injection was impulsive, while the variations in $\delta$ inferred from the microwave spectrum after 11:05 seem to be too small to account for the difference between the electron spectra in the flare HXR peak and main radio burst.

This source of the main burst was apparently different from the source of the first minor peak. With an almost constant electron spectrum index and nearly constant peak frequency during the main burst, its source must be static. The gradual changes in the peak frequency could be due to a varying number of emitting electrons and minor variations in the electron spectrum. This behavior does not support a possible association of the emitting source with either the $\mathrm{CME}$, whose flux-rope moved away from the solar surface up to $\approx 3 \mathrm{R}_{\odot}$ at 11:24, when the burst still continued, or a CME-related shock wave.

To summarize, the flare was comprised of two main acceleration episodes manifested in the HXR peaks observed by HEND. The first episode accounts for the first minor peak of the emissions observed from the Earth's direction. The second flare episode coincides with the onset of the main burst in all emissions observed from near-Earth vantage points. This burst was much longer than the flare HXR peak that suggests a possible confinement of emitting particles in a magnetic trap injected there during the second flare episode. The $>100 \mathrm{MeV}$ $\gamma$-ray burst shows a similar behavior to the HXR and GS burst, suggesting a common location of emitting electrons and protons. Plotnikov, Rouillard, and Share (2017) also concluded that accelerated electrons and protons had a common source. The spectrum of these particles was considerably harder than the second flare peak. If the second flare-acceleration episode supplied particles responsible for the main burst, then their additional acceleration was required. 


\subsection{Radio Sources}

Ackermann et al. (2017) showed that the radio burst was dominated by the gyrosynchrotron emission even in the metric range, at frequencies $>200-300 \mathrm{MHz}$. This made it possible to use observations from the Nançay Radioheliograph (NRH) in the analysis of the GS source presented by Carley et al. (2017). The authors revealed an off-limb GS source with a large extent centered above the flare position. The source appeared by 11:01 and remained centered at this position until about 11:05, expanding along the limb to occupy the position angles approximately from $50^{\circ}$ to $87^{\circ}$ at $11: 02$. This time interval corresponds to the first minor peak. After 11:05, the authors found a motion of the centroid position of the source southward with a speed of $\approx 1500 \mathrm{~km} \mathrm{~s}^{-1}$, while its height was almost unchanged. The authors related this GS source to the CME.

The hot blob in Figure 2 apparently corresponded to the CME's flux rope, being the most probable candidate for a CME-related GS source. However, the blob rose radially and did not exceed laterally a narrower range of position angles from $68^{\circ}$ to $78^{\circ}$ by $11: 02$. This behavior is incompatible with that of the radio source reported by Carley et al. (2017).

To understand the situation, we produced the images from 10-second integrated NRH data using the SolarSoft NRH package (secchirh.obspm.fr/nrhpackage.php) at $327 \mathrm{MHz}$ and $432 \mathrm{MHz}$ during 11:00-11:10 with an integration time and steps of 30 seconds. The images at both frequencies show that one nearly static source (GS1) appeared at about 11:01 and faded to about 11:05, when another static source (GS2) appeared. This source was located approximately above the Equator and had a lesser extent along the limb. GS2 was detectable until at least 11:10.

We did not consider a lateral expansion of GS1 that Carley et al. (2017) detected in their higher-resolution images. This expanding component resembles in behavior the EUV wave propagation (see the Electronic Supplementary Material AIA211_EUV_waves.mpg) and might be due to a possible Type II precursor continuum or another emission not related to relativistic electrons. Thus, the fast southward motion of the centroid position found by Carley et al. (2017) was most likely caused by a change in the brightness distribution among the two nearly static sources.

Neither GS1 nor GS2 exhibited any significant displacement, while their possible minor motions are beyond our scope. Figure 5 presents the contours of the NRH images averaged over the first minor peak duration for GS1 and over an interval of 11:05 - 11:10 for GS2 corresponding to a considerable part of the main burst, including its maximum. Each of the two sources considerably exceeded the NRH beam size; thus, the NRH images represent their realistic dimensions.

To reveal coronal structures, with which each of the two sources was associated, we invoke the coronal-dimming phenomenon. The CME lift-off rapidly stretches closed structures, leaving density depletion behind it. This process shows up as dimming, whose development is visible in the left panel of the AIA211_EUV_waves.mpg Electronic Supplementary Material. This panel in the movie presents the ratios of each current image to a fixed image observed before the event. A large dimmed area expanded in the movie. The dimming depth increased by about 11:10, and then the coronal-plasma density started recovering. 


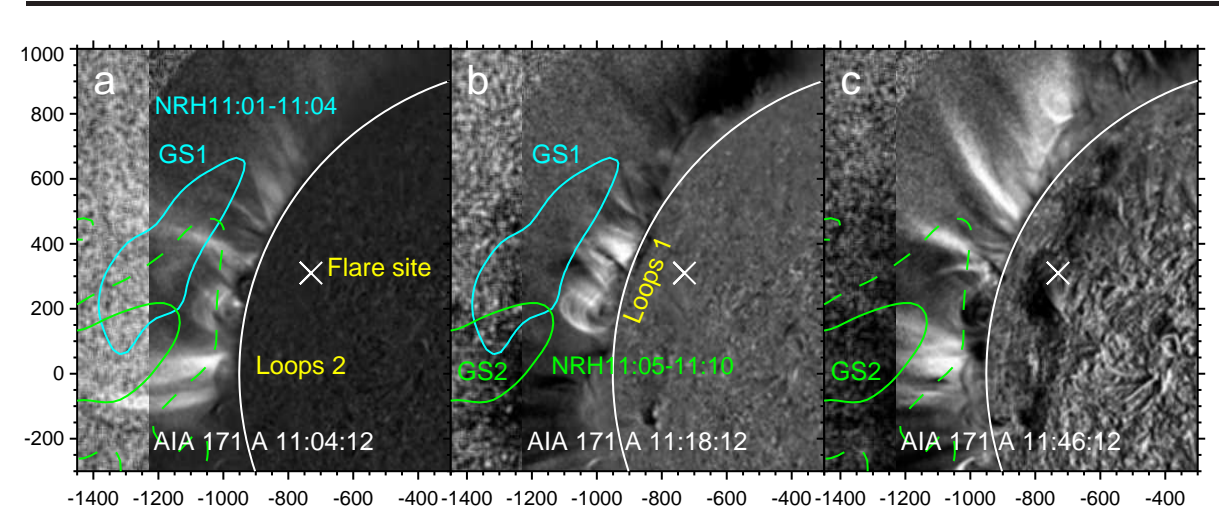

Figure 5. Coronal loops in AIA $171 \AA$ and SWAP $174 \AA$ images. Gyrosynchrotron sources observed by $\mathrm{NRH}$ at $432 \mathrm{MHz}$ are shown by the color contours. The blue contours represent the first source (GS1) at a level of 0.6 of its maximum brightness. The green contours represent the second source (GS2) at levels of 0.6 (solid) and 0.22 (dashed; panels a and c) of its maximum brightness. The slanted cross denotes the projected position of the flare site.

Dividing AIA images by the deepest-dimming image at 11:10 emphasizes the coronal structures that initially faded and then reappeared.

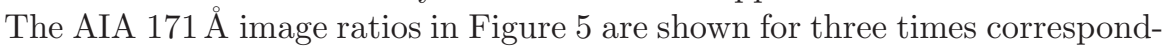
ing to the deepening of the dimming in Figure $5 \mathrm{~b}$ and its recovery in Figures $5 \mathrm{~b}$ and 5 . The field of view is extended to the left using $174 \AA$ images produced by the Sun Watcher using Active Pixel System detector and Image Processing (SWAP: Berghmans et al.,2006) onboard the Proba 2 micro-satellite.

Source GS1 was associated with an uppermost part of a far-side arcade denoted "Loops 1", whose top is visible above the limb. The near-the-limb portion of GS1 is most likely invisible because of refraction. Source GS2 was associated with different long loops denoted "Loops 2", which are deflected by the rising CME in Figure $5 \mathrm{~b}$ and relaxed to an equilibrium state in a much later Figure 5 . We did not analyze a possible small deviation in the position of GS2 that would correspond to the minor motions of the loops. No on-disk manifestations are visible in the NRH images, except for a lower-frequency $(\lesssim 300 \mathrm{MHz})$ static noise-storm source in the southern part of the Sun that was irrelevant to the eruptive event in question (Carley et al., 2017).

Thus, a static source GS1 was responsible for the first minor peak around 11:02:20. Carley et al. (2017) found that this peak was caused by the GS emission from electrons with a power-law index $\delta=3.2$ in an energy range from $9 \mathrm{keV}$ to $6.6 \mathrm{MeV}$ in magnetic field of $4.4 \mathrm{G}$ and an ambient plasma density of $n_{0}=1.3 \times 10^{8} \mathrm{~cm}^{-3}$. This power-law electron spectrum index is close to the semirelativistic approximation in our Figure \$, while the peak frequency in Figure 40 estimated from the San Vito data is somewhat lower than Carley et al. (2017) found from the Sagamore Hill fluxes reduced because of operational issues. The peak frequency here is strongly affected by the Razin suppression, which is determined by the ambient plasma density. It was depleted at this time because of the developing dimming; thus, the parameters estimated by Carley et al. (2017) are most likely correct with a reduced ambient plasma density. 
Another off-limb static source GS2 was responsible for the main long-duration burst. To estimate its parameters, we used the GX simulator of the GS emission (Kuznetsov, Nita, and Fleishman, 2011). The best fit of the actual radio spectrum near the maximum at 11:08 is reached with a magnetic-field strength of about $1 \mathrm{G}$, electrons with an index of $\delta \approx 2.8$, which lies between the two approximations in Figure 4 4 , a low-energy cutoff on the order of $100 \mathrm{keV}$ and an ambient density of a few $10^{8} \mathrm{~cm}^{-3}$. The simulations indicate that both bases of the emitting loops were most likely occulted; with an on-disk position for at least one of them, the fluxes around the peak frequency become flatter than the observations show.

There were no on-disk signatures of the GS emission. If it had been produced by returning electrons accelerated by a shock wave, which expanded away from the Sun (the scenario advocated by Plotnikov, Rouillard, and Share, 2017 and Jin et al., 2018), then the source should move over the solar surface, as Hudson (2017) pointed out. This situation is not observed.

In summary, the GS sources observed by NRH confirm the indications provided by the temporal profiles. Sources GS1 and GS2 were distinct, each of them was nearly static and located off-limb, and none of them was associated with the structures of the rising CME. Source GS1 emitted by closed loops was related to the first HXR flare peak and did not show any significant trapping. The longlived source GS2 appeared in a different, higher, closed structure during the second HXR flare peak, which probably initiated the main burst visible from the Earth's direction. The region of the GS2 radio source is also a most probable candidate for the long-duration HXR and $>100 \mathrm{MeV} \gamma$-ray emissions. In contrast to the first peak, the main burst is suggestive of a prolonged confinement of emitting particles in a magnetic trap.

\subsection{Coronal Configuration}

To analyze the coronal configuration, we compare the coronal loops observed in the EUV with magnetic-field lines extrapolated from photospheric magnetograms produced by the Helioseismic and Magnetic Imager (HMI: Scherrer et al., 2012) on SDO. We used the Potential Field Source Surface (PFSS) model from the SolarSoft package provided by the Lockheed Martin Solar and Astrophysics Laboratory (LMSAL: www.Imsal.com/ derosa/pfsspack/). Because the flare-hosting active region (AR) 12158 was located behind the east limb, extrapolation is only possible from a synoptic magnetogram. We used a magnetogram for Carrington Rotation 2155, in which AR 12158 was mapped about ten days after the event, being not yet present in the previous-rotation magnetogram. Analysis of open magnetic fields over a large part of the solar surface has led to the results very similar to those presented by Plotnikov, Rouillard, and Share (2017).

For the comparison we produced a combined image of coronal loops observed in EUV before the event. It is shown in Figure 6h. The main part of the image is an average over two AIA $171 \AA$ images divided by the azimuthally averaged radial brightness distribution (the technique is described in Kochanov et al., 2013). The field of view is extended by an enhanced-contrast average over 11 


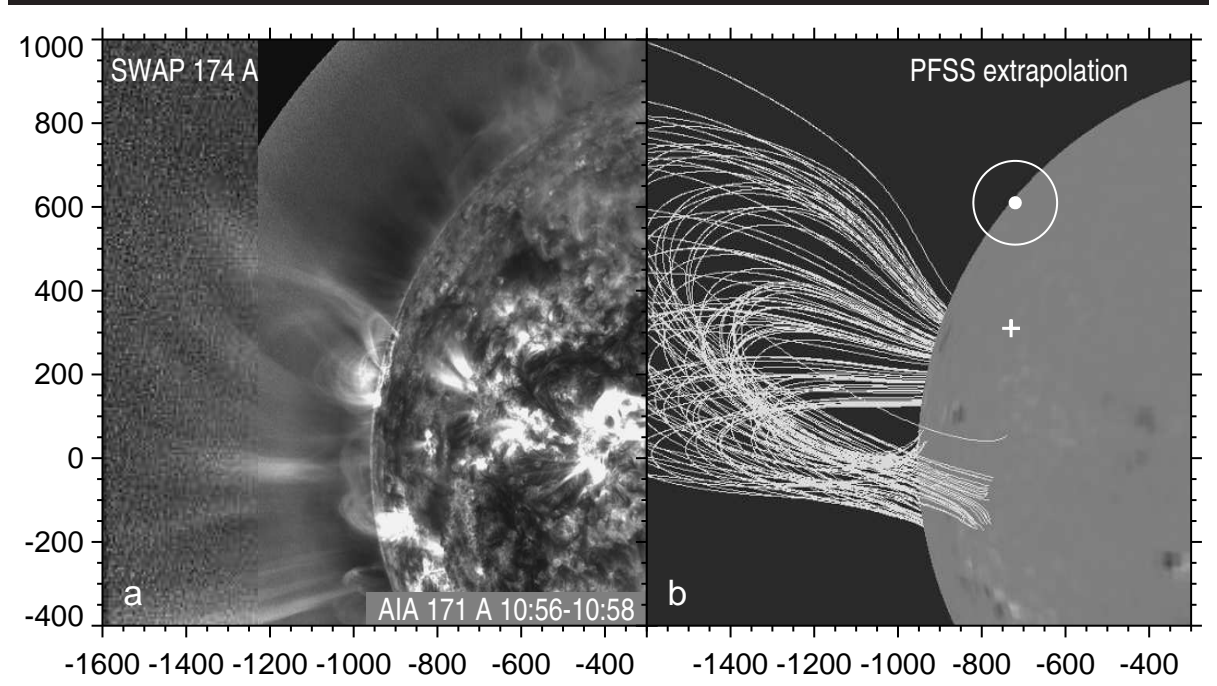

Figure 6. Coronal loops in a combined pre-event EUV image composed from AIA $171 \AA$ and SWAP $174 \AA$ images (a) and closed magnetic-field lines extrapolated from the flare site using the PFSS model (b). The cross denotes the projected position of the flare site. The dot denotes the Fermi /LAT $>100 \mathrm{MeV}$ emission centroid position with the $68 \%$ error circle (from Ackermann et al., 2017).

SWAP $174 \AA$ images observed from 10:31:21 to 10:55:12. Figure @b presents a set of loops extrapolated from a small region embracing the flare site.

The long loops visible in EUV near the limb close to the Equator (Figure 6a, $y \approx 0^{\prime \prime}$ ) are acceptably reproduced by the extrapolated-field lines in Figure 6 b. The lowest loops among the presented set are comparable in length with the solar radius, and the others are even longer. All of these loops emanate from the flare site. For some of them, the opposite ends are rooted behind the limb, and some others come to the visible side near the equator. Comparison with Figure 5 shows that the gyrosynchrotron source GS2 responsible for the main long-duration burst was located in these long loops. Their Earth-facing legs visible around $y \approx 0^{\prime \prime}$ produced a brighter radio emission.

The region of the Fermi/LAT $>100 \mathrm{MeV}$ centroid position and its wide environment were entirely covered by closed field lines over a wide range of altitudes. None of the field lines was connected with the flare site. Neither were there any open magnetic fields. To keep the figure decipherable, we do not show the field lines in this region located within a very large magnetic domain isolated from the domain in which the flare region resided.

The potential-field extrapolation used here (as well as force-free methods) is not able to reproduce the magnetic configuration during the CME eruption, which is the strongest violation of stationary conditions. The violation typically involves a nearby environment of the eruption region. In rare cases, which we call anomalous eruptions, reconnection occurs between an erupting structure and large-scale coronal environment (e.g. Grechnev et al., 2008a, 2011c, 2013b, 2014a; Uralov et al., 2014). Typical manifestations of an anomalous eruption are dispersal of the erupted material over a considerable part of the solar surface and 
microwave depressions ("negative bursts"). Such phenomena are best visible in the $304 \AA$ channel; in exceptional cases they are manifested in all EUV channels (e.g. the SOL2011-06-07 event: Grechnev et al.,2013b; van Driel-Gesztelyi et al., 2014). We examined all EUV channels of STEREO/EUVI and SDO/AIA on 1 September 2014 but have not found any manifestations of dispersed or returning erupted material. Neither there was any microwave depression. Thus, we have not found clear support from EUV or microwave observations to the scenario proposed by Jin et al. (2018). Furthermore, presumable reconnection in this scenario between the erupting structure and the domain, where the Fermi/LAT $>100 \mathrm{MeV}$ centroid was located, had to proceed very deep into the closed-field area to reach the connection to the photosphere.

\section{Shock Waves}

The presence of a shock wave in this event is indicated by a high speed measured for the leading edge of the CME in the online CME catalog (cdaw.gsfc.nasa.gov/CME_list/ Yashiro et al., 2004). Plotnikov, Rouillard, and Share (2017) measured some of the shock-wave characteristics based on three-dimensional reconstructions of the wave front from EUV and coronagraph observations. A shock wave can also be manifested in a Type II burst and EUV wave. As shown previously (e.g. Grechnev et al., 2008a, 2011a, 2016, 2017, 2018), these signatures can be reconciled kinematically with each other and with a halo embracing a fast CME. Here we consider the shock-wave traces observed in EUV and coronagraph images and possible shock signatures in a dynamic radio spectrum.

\subsection{Shock-Wave Signatures in EUV and Coronagraph Images}

Figure 7 and the Electronic Supplementary Material AIA211_EUV_waves.mpg present EUV wave propagation observed in AIA $211 \AA$ images separated by two minutes. Figure 7 and the right panel of the movie show contrasted running differences. The left panel of the movie shows the ratios of each current image with a fixed pre-event image observed at 10:56, in which solar rotation was compensated for to the time of the current image. Such ratio images are free from spurious effects in running-difference images caused by subtraction.

An off-limb brightening in Figure 7 facing the far-side flare region denoted by the dot suggests that something already happened as early as 10:58. As Figure2 indicates, this brightening was due to expansion of high coronal loops caused by an erupting structure, whose lift-off commenced at that time. The EUV wave appears in Figure 7b at 11:00. Its front is indicated in all images by the white arrow parallel to the limb and by the blue bar in the movie. About two minutes later, the second EUV wave front appears (black arrow and red bar). Being present in non-subtracted ratio images, it cannot be an effect of subtraction.

The northern flanks of the EUV waves manifest in a bright compression region. The expansion at the southern flank is also detectable, but without a clear leading brightening; it looks like a deepening of an expanding dimming.

We measured the motion of the northern EUV waves' leading edges at a fixed distance from the limb. The images shown in Figure 7 are sampled uniformly in 


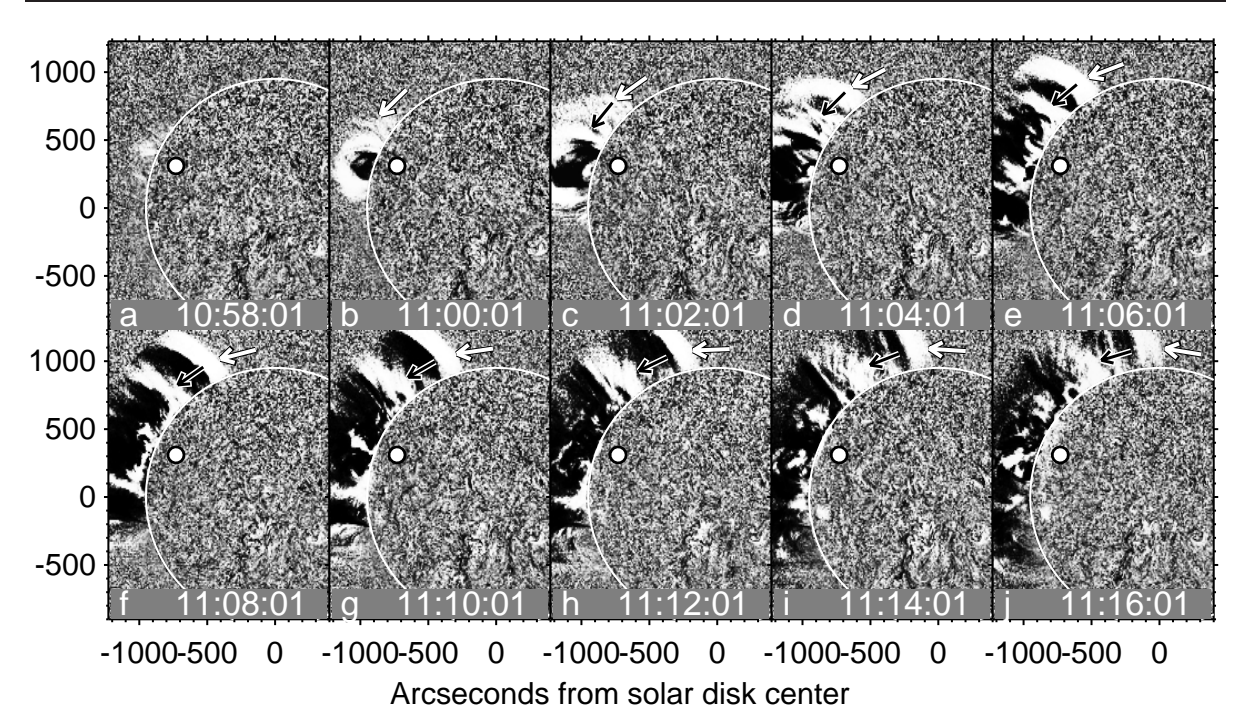

Figure 7. EUV wave propagation along the limb observed in running-difference AIA $211 \AA$ images. The arrows point at the first (white arrow) and second (black arrow) EUV wave fronts. The white dot denotes the projected position of the flare site. The axes show the distances from solar disk center in arcseconds. The temporal interval between all consecutive images is two minutes.

steps of two minutes. The positions of the white-arrow head indicate that the plane-of-the-sky EUV wave speed along the limb was highest initially and then monotonically decreased. For example, the EUV wave speed in Figure $7 \mathrm{~b}$ (11:00) was $\approx 1000 \mathrm{~km} \mathrm{~s}^{-1}$, and in Figure 7 (11:16) it decreased to $\approx 560 \mathrm{~km} \mathrm{~s}^{-1}$. Using a power-law fit to the measurements as described in our articles listed in Section 3 , we estimated the onset time for the first EUV wave $t_{01}=10: 59: 04 \pm 15$ seconds. It is more difficult to identify and measure the second EUV wave front. Its probable onset time is $t_{02}=11: 02: 00$ with an uncertainty being presumably within one minute. The measurements are presented and discussed in Section 3.3. the wave speeds are shown in the bottom panel of the movie by the corresponding colors. Both EUV waves decelerated. Deceleration of EUV wave 2 was weaker, which is not obvious from the plot, because the strongest-deceleration initial part of the faster EUV wave 1 is not shown.

The EUV waves propagated over a huge area. In Figure 7], the projected northern flank of EUV wave 1 reached the North Pole, while the southern flank reached the lower edge of the image shown. These moving features apparently had a wave nature.

The expanding wave dome was also observed by STEREO-B (here we focus on the first wave). Figures 8 a -8 present it in combined COR1 and EUVI $195 \AA$ running-difference images. As Plotnikov, Rouillard, and Share (2017) showed, the shape of the wave front was close to an ellipsoid (using the same method, Rouillard et al. (2016) made a similar conclusion for a different event). Here we did not pursue to catch the wave-dome shape; instead, the black-on-white circles in Figures 8 - 8 ; approximately reproduce its size. The correspondence between the outlining circles and observations is almost perfect in Figures $8 \mathrm{a}$ and 
8b. In other panels, thick apparent flanks dominate, being probably emphasized by deflected streamers and subtraction of the images. Nevertheless, the circles correspond to the faint outermost EUV wave signatures on the solar disk. These circles corresponding to a fixed projection of an expanding ellipsoid, whose shape does not change considerably ( $c f$. Grechnev et al., 2011b). Thus, the circles correctly reproduce the kinematics of the wave-dome expansion, differing from the highest-speed direction by a nearly constant factor. The measurements are presented in Section 3.3

Figures $8 \mathrm{~g}-8$ present enlarged EUVI $195 \AA$ running-difference images corresponding to Figures $8 \mathrm{a}-8 \mathrm{r}$. Figure $8 \mathrm{~b}$ reveals a loop-like structure denoted "Loop" probably corresponding to the lower part of the loops shown in Figure 6. in which gyrosynchrotron source GS2 was located. Figures 8h and 8 show the EUV wave indicated by the horizontal arrow that corresponds to Figure7 7 viewed from a near-Earth vantage point. The lower part of the wave front is tilted with respect to the solar surface. Figure 8 reveals three to five streamer-like structures highlighted by the wave passage.

\subsection{Dynamic Radio Spectrum}

A dynamic spectrum in Figure 9 presents a radio burst at $10-180 \mathrm{MHz}$ produced by this event. The spectrum was composed from data of the Nançay Decametric Array (NDA: Lecacheux, 2000) at $10-80 \mathrm{MHz}$ and data of the spectrographs at the Sagamore Hill $(80-128 \mathrm{MHz})$ and San Vito $(128-180 \mathrm{MHz})$ RSTN stations. The dynamic spectrum presents emissions generated at different locations. The structure of the burst is complex and contains unusual features. Identifying Type II bands that carry information about a shock wave is complicated by a series of stronger Type IIIs (Type VI), a gap between 85 and $110 \mathrm{MHz}$, and interferences at higher frequencies. To search for indications of possible Type II bands, we plotted their expected trajectories on top of the dynamic spectrum and, adjusting their parameters, we tried to fit them to presumable Type II signatures.

The method to calculate a trajectory of a Type II burst is described in our preceding studies (e.g. Grechnev et al., 2011a, 2017). We use a power-law density model $n(x)=n_{0}\left(x / h_{0}\right)^{-\mu}$ where $x$ is the distance from the eruption center, $n_{0}=5.5 \times 10^{8} \mathrm{~cm}^{-3}$ is the density at a distance $h_{0}=100 \mathrm{Mm}$ (close to the scale height), and $\mu$ is the density falloff exponent. This model with appropriate parameters is close to the Saito model (Saito et al., 1970) in the far zone, where the corona is quiet (Section 3.3), and provides higher densities in the near zone $(<260 \mathrm{Mm})$, where the corona is strongly disturbed by the eruption. Referring to an arbitrary point on the dynamic spectrum at time $t_{1}$, we choose a frequency and calculate a corresponding distance $x_{1}$ from our density model for the first or second harmonic of the plasma frequency. The wave onset time $t_{01}=10: 59: 04$ was estimated from AIA data. Then, we calculate the Type II trajectory as $x(t)=x_{1}\left[\left(t-t_{01}\right) /\left(t-t_{1}\right)\right]^{2 /(5-\mu)}$. A similar approach was used in Section 3.1 to measure the shock-wave kinematics from its signatures in AIA, EUVI, and COR1 images. 

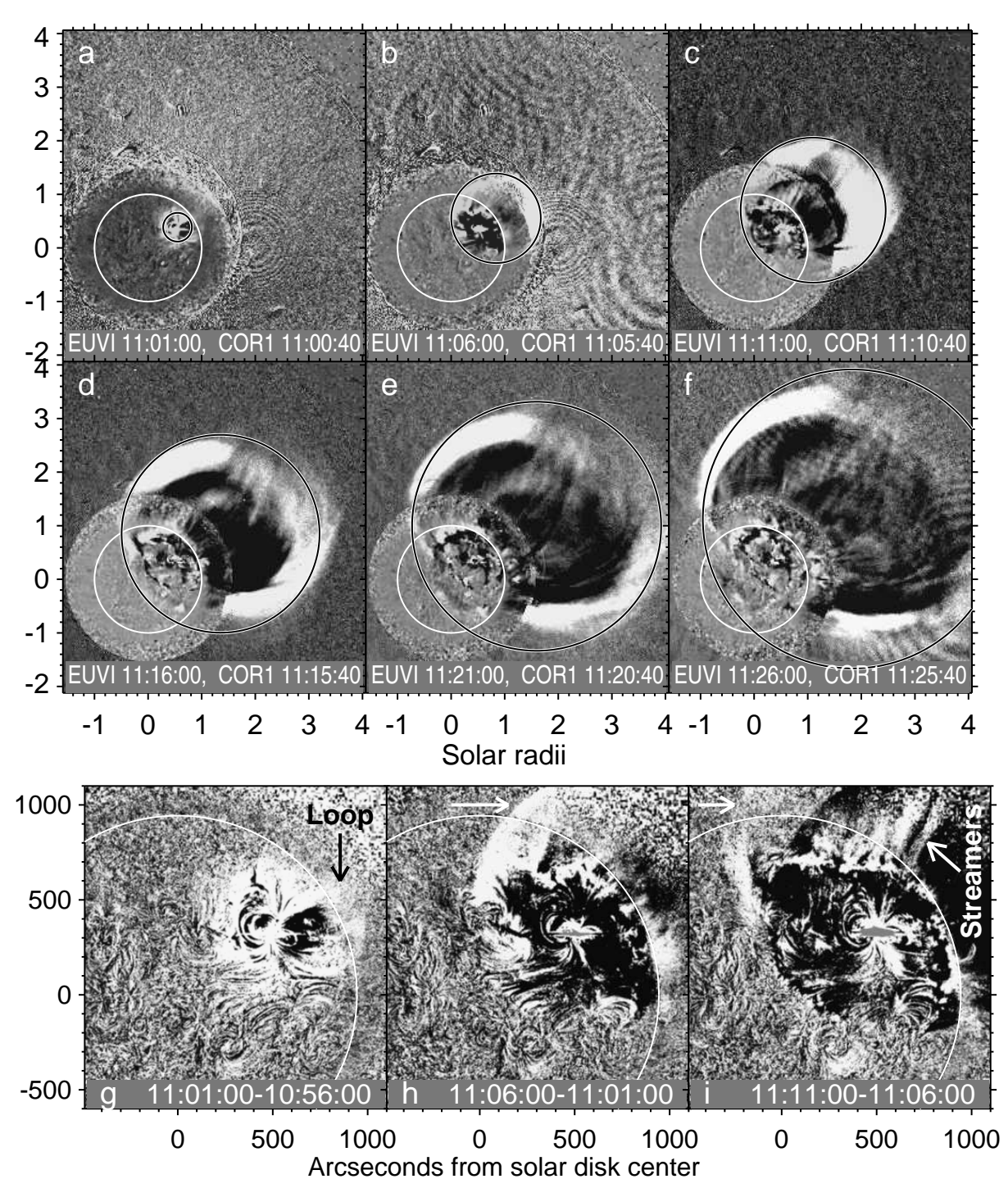

Figure 8. a-f) Wave traces observed by STEREO-B/COR1 and EUVI $195 \AA$ running-difference images. The black-on-white circles approximately reproduce the extent of the wave front. The white circles denote the limb. $\mathrm{g}-\mathrm{i}$ ) Wave signatures in enlarged EUVI $195 \AA$ running-difference images shown in panels a-c. Panel g reveals a set of long loops ("Loop"). The horizontal arrows in panels $\mathrm{h}$ and $\mathrm{i}$ indicate the EUV wave above the limb. Panel i reveals a set of streamer-like structures.

The dotted and dashed curves plotted in Figure 9 are harmonically related (2:1) and correspond to an expected trajectory of a Type II burst produced by the first shock wave with an onset time $t_{01}=10: 59: 04$ in plasma with a density falloff exponent $\mu=2.75$. The trajectories more or less correspond to slowly drifting features 1 and 2 discernible between 11:02:40 and 11:05:00, al- 


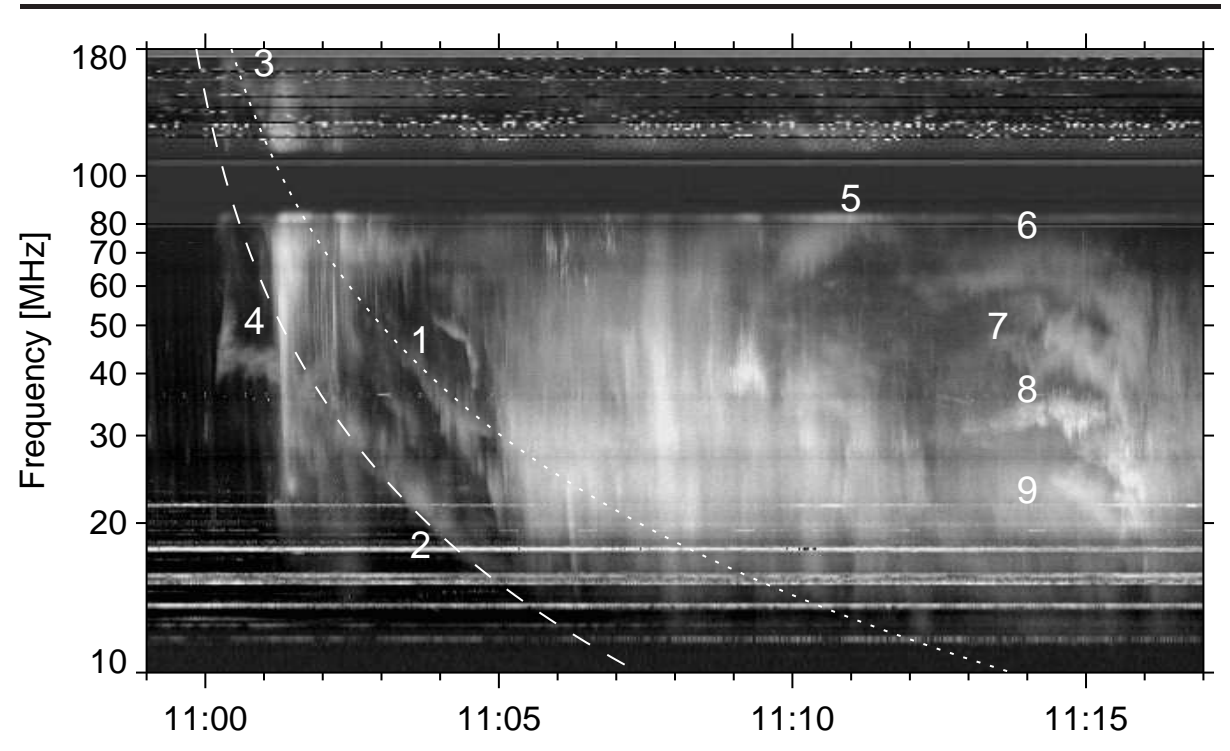

Figure 9. Combined dynamic spectrum of the radio burst composed from the NDA, Sagamore Hill, and San Vito data. The harmonically related dotted and dashed curves correspond to an expected trajectory of a Type II burst produced by a shock wave with an onset time $t_{01}=10: 59: 04$ in plasma with a density falloff exponent $\mu=2.75$. Labels 1,2 , and 3 denote slowly drifting features that might be possible Type II bands. Non-drifting feature 4 with a reversely drifting onset is also a possible Type II-like manifestation. Features $5-9$, some with reverse drifts, might also be due to Type II emissions from different structures.

though their structures are different. The corresponding kinematics is presented in Section 3.3

The drift rate of the faint higher-frequency feature 3 is somewhat different from the calculated trajectories and can be reproduced by using a lesser $\mu \approx 2.3$. The source of this emission was probably located in a different structure.

A narrow-band $(\approx 10 \%)$ feature 4 starts from a fast reverse drift and does not exhibit any drift afterward. Its onset suggests an interaction of a quasiperpendicular shock on an extended structure with a contact point rapidly moving to its base. This feature might be caused by a collision of a curved first shock front with a long loop. The collision could excite wave processes responsible for the emission at the plasma frequency in the loop.

A set of unusual features $5-9$ is visible between 11:10 and 11:16. They start from reverse drifts, which later turn to the normal direction from high to low frequencies. These features are relatively narrow-band and have Type III-like structure, similar to Type II bursts. A harmonic counterpart to feature 5 is not detectable. The pairs $(6,8)$ and $(7,9)$ are both harmonically related, although the structures of the bands in each pair are not identical. Features 5-9 might also be due to Type II emission produced by the passage of the shock wave, but the cause of their unusual drifts is not obvious. Their spectral evolution is different from nondrifting Type II-like bursts presented by Aurass, Vršnak, and Mann (2002) and Aurass (2003) and from inverse-N-like shifts of Type II bands (Grechnev et al., 2011a, 2014b). 
As demonstrated in our preceding studies (e.g. Grechnev et al., 2015, 2016, 2018), the most probable source of a narrow-band Type II emission is a streamer. The shock crossing the streamer deforms the plasma flow in the vicinity of its current sheet that induces a flare-like process running along the streamer together with the intersection point. Figures $8 \mathrm{r}$ and 8 reveal a set of small streamers visible indeed in an EUVI image at 11:11. The streamers appeared, being blown by a shock front. An oblique shock and compressed plasma flow behind it displace plasma in the streamer along it. The effect is strongest near the shock normal and decreases away from it. Thus, just after the passage of the first shock, an inverse density distribution forms for some time in a portion of the streamer. When the second shock hits the streamer about three minutes later, the intersection point moves along the streamer up. The instantaneous drift rate reflects the distorted density distribution in the streamer caused by the passage of the preceding shock.

The exact number of the streamers in Figure 8 is not obvious, and neither is the exact number of the sources responsible for features 5-9 in Figure 9, In addition, the presence of a streamer is a necessary but not sufficient condition to produce Type II emission. With these uncertainties, the number of the streamers roughly corresponds to the response in the dynamic spectrum.

\subsection{Summary on Shock-Wave Measurements}

The measurements in Sections 3.1 and 3.2 were fitted using power-law distancetime relations: $x(t) \propto\left(t-t_{01}\right)^{2 /(5-\mu)}$. Power-law kinematic plots of a shock wave shown on a $\log -\log$ scale with the origin of the time axis at the wave onset time $\left[t_{0}\right]$ are represented by straight lines. We used in Figure 10 the same $t_{01}=10: 59: 04$ in all cases and density falloff exponents $\mu=2.75$ for the wave signatures in COR1 and EUVI images and dynamic spectrum; $\mu_{\mathrm{AIA}}=2.51$ for EUV wave 1 , and $\mu_{\mathrm{AIA}}=2.75$ for EUV wave 2 , both running along the limb in AIA images (Figure 7). The plot for EUV wave 2 is conspicuously curved, because it started three minutes after the origin of the plot. This situation demonstrates the sensitivity of the $\log -\log$ representation to the wave onset time, which permits one to estimate it with a high accuracy.

Figure 10 presents the results of the kinematical measurements and their analytic fit by different line styles. Our measurements exactly coincide with the fitting lines, because the outlining curves in Figures 7,8 , and 9 were calculated analytically and adjusted to the observed wave signatures.

The data from the online CME catalog are shown by asterisks for the measurements from LASCO-C2 images and by diamonds for those from C3 images. The gray squares denoted "PRS" represent the measurements by Plotnikov, Rouillard, and Share (2017). All of these heliocentric distances are reduced by $0.84 \mathrm{R}_{\odot}$ to refer to the eruption site (assumed to coincide with the flare position).

The measurements in the CME catalog reveal kinematics close to our results in Figure 10 $\mathrm{k}$. The dotted line calculated as our fit of the measurements from EUVI and COR1 images magnified by a factor of 1.4 acceptably matches the data from the CME catalog up to $10 \mathrm{R}_{\odot}$. 

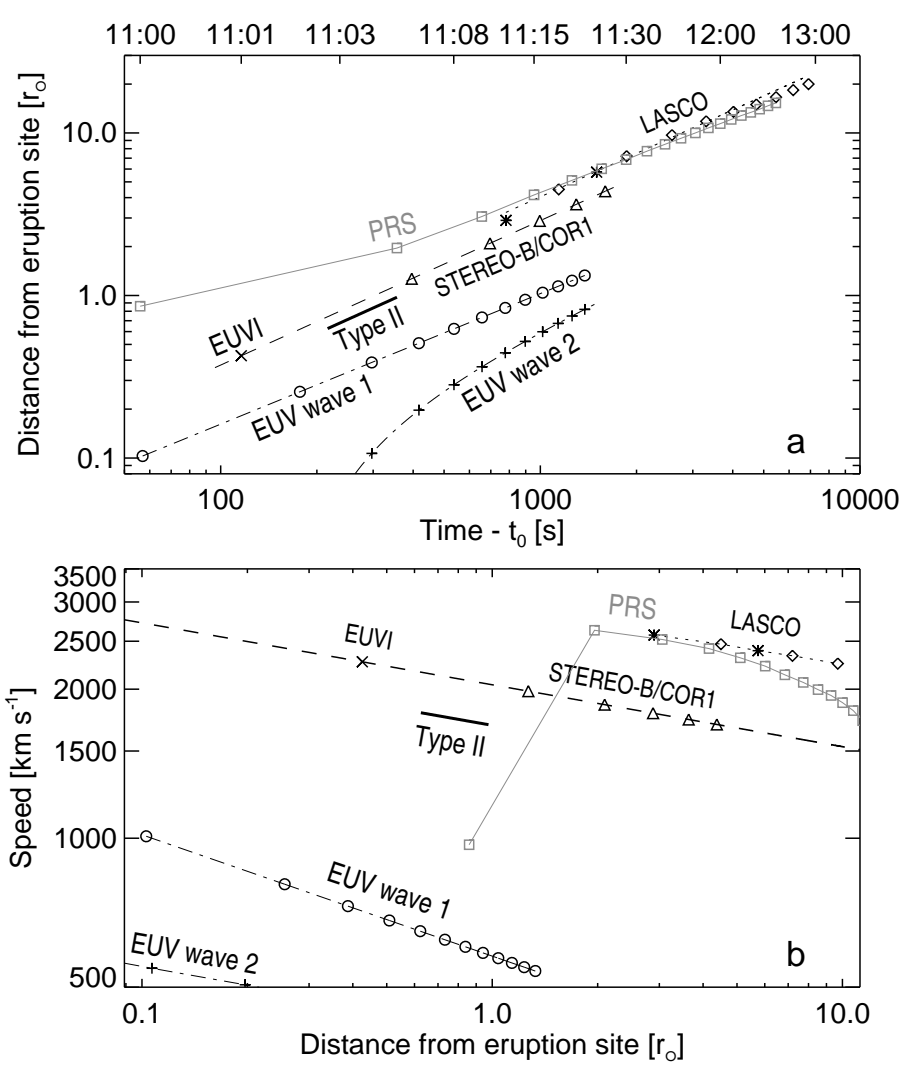

Figure 10. Decelerating power-law kinematics of the shock waves measured from different vantage points using different methods. All distances are measured from the eruption center located at $0.84 \mathrm{R}_{\odot}$. a) Distance-time plots. The horizontal axis represents time after the wave onset $t_{01}=10: 59: 04$ in the logarithmic scale. The upper $X$-axis shows actual times. b) Speed-distance plots. The symbols represent our measurements from the images produced by different telescopes. The measurements from the CME catalog are shown by asterisks (C2) and diamonds (C3). The thick bar represents the shock kinematics corresponding to possible Type II bands outlined in the dynamic spectrum. The gray squares denoted "PRS" represent the measurements from Plotnikov, Rouillard, and Share (2017). The black lines of different styles show the analytic fit.

All measurements in Figure 10a present similar distance-time histories, except for the curved plot for EUV wave 2 because of its later wave-onset time: $t_{02}>t_{01}$. The difference of $40 \%$ between the measurements in the CME catalog from LASCO images and our measurements from STEREO images may be caused by the ellipticity of the shock-wave dome and still more probably by the different techniques used. The difference within $20 \%$ between the measurements from COR1 images and dynamic radio spectrum can reflect the difference in the shock-wave propagation directions and in plasma densities in coronal structures. The difference between the measurements from AIA and COR1 images reflects a slower motion of the EUV wave over the solar surface and its stronger deceleration with respect to the wave dome expanding away from the Sun (Figures $8 \mathrm{~b}-8 \mathrm{f})$. 
The slopes of the straight fitting lines are $2 /(5-\mu)$ corresponding to $\mu=2.51$ (the slope of 0.80 ) for EUV wave 1 and to $\mu=2.75$ (0.89) for all others. The power-law density model $n(x)=n_{0}\left(x / h_{0}\right)^{-\mu}$ with $n_{0}=3.75 \times 10^{8} \mathrm{~cm}^{-3}$ and $\mu=2.75$ is close to the Saito model (Saito et al., 1970) at the latitude of the flare site $14^{\circ}$ within $\pm 30 \%$ at distances from $260 \mathrm{Mm}$ to $25 \mathrm{R}_{\odot}$. Recall that we use $n_{0}=5.5 \times 10^{8} \mathrm{~cm}^{-3}$ and measure the distance $[x]$ from the eruption center, while the Saito model refers to the heliocentric distance $r=R / \mathrm{R}_{\odot}$, so that $x \approx(r-1) \mathrm{R}_{\odot}$ in the radial direction. Overall, the measurements made using different methods are in a reasonable agreement with an expected propagation of a decelerating blast-wave-like shock in a typical corona.

The expected speed-time dependencies can be obtained by differentiation of distance-time plots, $v(t) \propto\left(t-t_{0}\right)^{2 /(5-\mu)-1}=\left(t-t_{0}\right)^{(\mu-3) /(5-\mu)}$. The shock wave propagating in plasma with a density falloff exponent $\mu<3$ monotonically decelerates. We only observed decelerating shock waves so far.

For the speed vs. distance dependence we get from the analytic fit $v(x) \propto$ $x^{(\mu-3) / 2}$. In this event, the slope of the speed-distance plots is $-0.13(\mu=2.75)$ for most shock-wave signatures and $-0.23(\mu=2.54)$ for EUV wave 1 . The latter value corresponds to a stronger deceleration of the slower shock-wave trail on the solar surface in Figure 8, while the whole wave dome expanded self-similarly.

The speed-distance plots are shown in Figure 10p for the distances from $70 \mathrm{Mm}$ to $10 \mathrm{R}_{\odot}$ from the eruption site. Our measurements from different data agree with each other and with the measurements in the CME catalog within this range. The results of Plotnikov, Rouillard, and Share (2017) within (1.5 $5) \mathrm{R}_{\odot}$ are close to the measurements in the CME catalog, but they show a stronger deceleration at larger distances. However, the most challenging is their first data point with about three times lower speed than expected, whereas our measurements show the slope persisting down to much lower distances. The shock wave did not have any acceleration phase. Instead, the wave started from the fast-mode speed in its origin and monotonically decelerated. We have to conclude that the estimate by Plotnikov, Rouillard, and Share (2017) of the shock-wave speed at its earliest appearance is questionable.

Our results also disagree with Jin et al. (2018), who found the shock speed to increase from $\approx 400 \mathrm{~km} \mathrm{~s}^{-1}$ to $\approx 1000 \mathrm{~km} \mathrm{~s}^{-1}$ from the tenth minute since the eruption until the thirtieth minute. This time interval corresponds to the measurements from STEREO-B/COR1 in Figure 10, where the shock speed monotonically decreases from $\approx 2000 \mathrm{~km} \mathrm{~s}^{-1}$ to $\approx 1700 \mathrm{~km} \mathrm{~s}^{-1}$. A possible cause of the questionable result of Jin et al. (2018) might be the difficulty to identify the shock front from MHD simulations that the authors made. The incorrect behavior of the shock speed probably affected the derived plots. We hope our results can help in elaborating the promising method developed by the authors.

\section{Discussion}

\subsection{Trapping}

One of the challenges of this event is a contrast between the impulsive temporal profiles of the flare HXR emission observed by HEND and long-lasting 
gyrosynchrotron, HXR, and $\gamma$-ray emissions observed from the Earth's direction. Long-duration $\gamma$-rays have been observed in the past in a few events and extensively discussed (see, e.g., Forrest et al., 1985; Akimov et al., 1996; Ryan, 2000; Kurt et al., 2010; Kuznetsov et al., 2011). One of the possible explanations considered was long-term trapping of high-energy protons in closed coronal loops. Microwave bursts often exhibit manifestations of trapping of accelerated electrons (e.g. Melnikov and Magun, 1998; Silva, Wang, and Gary, 2000; Kundu et al., 2001). Trapping of protons might also occur (e.g. Mandzhavidze and Ramaty, 1992). The conditions to contain trapped relativistic protons or ions for a long time were summarized by Ryan (2000): low density, low turbulence, and force-free field. These requirements can be hard for flare loops but not critical for lower-density long loops like those in Figure [and high-energy protons responsible for the pion-decay emission.

\subsubsection{Temporal Profiles}

We ask if the trapping effect can produce the observed long-duration time profiles in response to particle populations injected impulsively. Figure 11 reproduces the HXR and $\gamma$-ray temporal profiles observed from different vantage points that were presented in Figure 3. As noted, the first HXR peak observed by HEND around 11:02:20 had a very close response in gyrosynchrotron and lower-energy hard X-rays. The main long-duration radio and HXR bursts represent the only response to the second HEND peak around 11:04:30.

At the first step, one should separate from the actual temporal profile the second HXR peak, which possibly was responsible for the long-lasting emissions observed from the Earth's direction. An appropriate shape has a function $\Psi(t, \mu, \tau)=t^{\mu} \exp (-t / \tau)$ (Aschwanden, 2004b) similar to the Landau probability distribution. To separate the second HXR peak, decomposition of the whole HXR temporal profile is required. We considered three peaks: a minor peak around 11:00:00, the first peak, and the second peak. The decomposition was made by least-squares fitting a linear combination simulating the three peaks to the $\operatorname{HXR}(t)$ temporal profile actually observed by HEND. Specifically, we minimized the average value $\bar{Q}$ of the $Q$ quantity defined as

$$
Q=\left[\operatorname{HXR}(t)-a_{0} \Psi\left(t, \mu_{0}, \tau_{0}\right)-a_{1} \Psi\left(t, \mu_{1}, \tau_{1}\right)-a_{2} \Psi\left(t, \mu_{2}, \tau_{2}\right)\right]^{2} .
$$

The dotted line in Figure 11b shows the actual HXR temporal profile. The solid line represents the net second peak evaluated from the decomposition.

At the second step, the emissions from trapped particles were simulated following the approach used by Kundu et al. (2001). The temporal profiles $[I(t)]$ were calculated as

$$
I(t)=\int_{-\infty}^{t} \exp \left[-\left(t-t^{\prime}\right) / \tau_{\text {trap }}\right] f_{\text {inj }}\left(t^{\prime}\right) \mathrm{d} t^{\prime},
$$

where the net second peak found at the first step was used as an injection function $\left[f_{\text {inj }}\right]$. The trapping times $\left[\tau_{\text {trap }}\right]$ were adjusted to make the temporal profiles simulated more or less similar to those actually observed. The results 


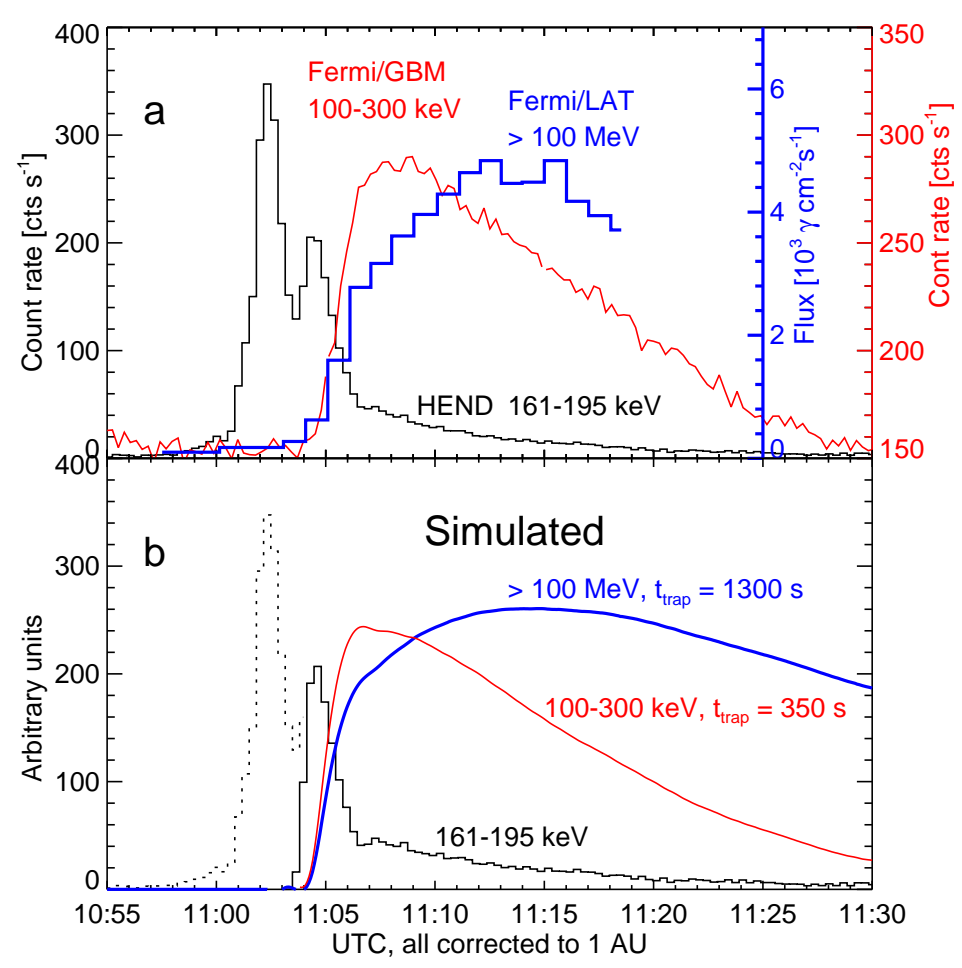

Figure 11. The trapping effect issue. a) Hard emissions actually observed (corresponding to Figure 3): $161-195 \mathrm{keV}$ flare hard X-rays (Mars Odyssey/HEND, black), $100-300 \mathrm{keV}$ viewed from the Earth's direction (Fermi/GBM, red), and $>100 \mathrm{MeV} \gamma$-ray burst (Fermi/LAT, thick-blue). The one-second and four-second time-resolution Fermi/GBM data are both summed over 16 seconds for convenience. b) Simulated temporal profiles expected as a result of trapping: the injection function for the second HXR peak $\left[f_{\text {inj }}\right]$ (black-solid), actual HXR temporal profile (black-dotted), and simulated emissions from trapped electrons (red) and protons (thick-blue).

are shown in Figure 11b by the curves, whose colors correspond to temporal profiles in Figure 117. The simulated temporal profiles acceptably reproduce the shapes of the bursts actually observed by Fermi in HXR and $\gamma$-rays. We remind the reader that the gyrosynchrotron radio burst was almost identical to the HXR burst in shape (Figure 3). Thus, the simulation demonstrates that the long-lasting HXR and radio emissions observed from the Earth's direction can well be accounted for by trapping of emitting electrons in closed coronal loops.

With a static position of the gyrosynchrotron source shown by NRH to be associated with long, closed loops, its origin due to emission from trapped electrons appears to be natural. The same is most likely related to the HXR burst, contrary to the idea of Plotnikov, Rouillard, and Share (2017) about the shockrelated origin of accelerated electrons high in the corona and their return to the solar surface along open magnetic structures. In any case, neither electrons nor protons have access into closed loops. 


\subsubsection{Low-Energy Cutoff of the Electron Spectrum}

An additional support to the role of trapping in this event is provided by the spectral information. According to Carley et al. (2017), the low-energy cutoff of the electron spectrum in the first peak during 11:01-11:04 was as low as $9 \mathrm{keV}$. For the main burst between 11:06 and 11:16, Plotnikov, Rouillard, and Share (2017) estimated the low-energy cutoff to be much higher, at $130 \mathrm{keV}$. We obtained a low-energy cutoff of order $100 \mathrm{keV}$ by fitting the gyrosynchrotron spectrum near the maximum of the burst at about 11:08.

The temporal behavior of the electron energy density spectrum with an increasing low-energy cutoff is really expected in a trap after an initial impulsive injection, $\Delta t_{\text {inj }} \ll \tau_{\text {trap }}$, of electrons with a power-law spectrum (Melrose and Brown, 1976; Metcalf and Alexander, 1999). For the estimate we use a formula from Melrose and Brown (1976) for the turnover energy $E_{\mathrm{T}}=\left(3 / 2 \nu_{0} t\right)^{2 / 3}$ of the spectrum of fast trapped electrons precipitating into the loss cone because of Coulomb collisions, where $\nu_{0}=5 \times 10^{-9} n_{0}\left[\mathrm{~s}^{-1}(\mathrm{keV})^{3 / 2}\right]$ and $n_{0}$ is the number density of thermal electrons. With an impulsive injection at 11:05 and an ambient density of $n_{0}=6.4 \times 10^{8} \mathrm{~cm}^{-3}$, we estimate $E_{\mathrm{T}}$ to be $91 \mathrm{keV}$ at $11: 08,44 \mathrm{keV}$ at $11: 06$, and $216 \mathrm{keV}$ at $11: 16$. The average turnover energy $E_{\mathrm{T}}$ between its values at 11:06 and 11:16 is $130 \mathrm{keV}$. These values expected for the spectrum of trapped electrons match the low-energy cutoffs estimated from observations. Note that our simplified simulations and estimates in Section 4.1.1 and the present section do not consider possible changes in the plasma density or other complications.

\subsubsection{Trapping of Protons}

The temporal profiles and spectral characteristics of the gyrosynchrotron and HXR emissions viewed from the Earth's direction are consistent with a longterm trapping of an electron population impulsively injected during the second peak. As our simulation shows, trapping of protons responsible for the pion-decay $\gamma$-ray emission was also a likely cause of its long duration. The $>100 \mathrm{MeV} \gamma$-ray temporal profile obtained in our simulation acceptably matches the actual light curve. The difference between the durations of the HXR/radio and $\gamma$-ray bursts observed from the Earth's direction is much less than the difference between either of them and the probable injection function. Plotnikov, Rouillard, and Share (2017) also concluded that accelerated electrons and protons responsible for the long-lasting emissions in this event had a common origin, contrary to the impression of Jin et al. (2018) about the drastic difference between the > $100 \mathrm{MeV}$ light curve and all other emissions. The long-lasting $\pi^{0}$-decay emission observed in a few solar events was previously considered by Mandzhavidze and Ramaty (1992) as evidence for trapping of particles in solar flares.

The trapping time has extensively been discussed in the literature (see Aschwanden, 2004a for a review and details). The factors determining the trapping times of electrons and protons in this event need a separate study.

\subsubsection{Appearance of Accelerated Particles in a Trap}

While the long-term trapping of electrons and protons in the long static set of loops associated with the GS2 source appears to be very probable, this set of 
loops did not exhibit direct participation in either the flare or CME formation. This circumstance raises a question of how high-energy particles came to these magnetic structures disconnected from the flaring structures and CME.

Most likely, flare-accelerated particles escape into interplanetary space or enter magnetic traps (static or moving) due to reconnection processes. Displacement of particles across magnetic field lines because of drifts or collisions occurs slowly and not efficiently; otherwise, trapping would be exceptional in solar events, contrary to observations.

In our view, the development of an eruption and flare usually starts within a bipolar configuration, which can be a part of a more complex magnetic structure, e.g. a magnetic domain of a quadrupole configuration. In this situation, four domains share one null point. The eruptive flux-rope carrying trapped flareaccelerated particles moves toward this point. The passage of the flux-rope at the null inevitably results in local reconnection between its magnetic flux and fluxes belonging to adjacent domains. As a result, both open and closed structures of these domains become filled with energetic particles as well as cool plasma of the pre-eruptive filament. Related schemes containing a single null point were discussed by Gary and Moore (2004), Masson, Antiochos, and DeVore (2013), Meshalkina et al. (2009), Grechnev et al. (2013b), and Uralov et al. (2014). Stretching a large-scale quadrupole into the solar wind might cause disappearance of the null point. Nevertheless, lateral reconnection between the flux-rope and coronal rays also occurs in this situation (e.g. Bemporad et al., 2010). The presence of a coronal null in the parent active region 12158 is supported by an $\mathrm{S}-\mathrm{N}-\mathrm{S}-\mathrm{N}$ configuration discernible in SDO/HMI magnetograms observed one week after the event.

\subsection{Position of the Gamma-Ray Source}

The $>100 \mathrm{MeV}$ emission centroid position calculated by Ackermann et al. (2017) from Fermi/LAT data with a $68 \%$ error radius of $100^{\prime \prime}$ is located at the east limb at a latitude of about N41 (Figures 1b and 1 ). As noted in Section 2.4, this site and its wide environment were totally covered by closed magnetic fields. Protons and other charged particles did not have access into this domain from either the flare region or larger coronal heights along open field lines. On the other hand, the off-limb radio source GS2, which is the most probable candidate for the source of long-duration HXR and $\gamma$-ray emissions, was located nearly above the Equator, far away from the Fermi/LAT $>100 \mathrm{MeV}$ emission centroid position. No other candidate for the source of the long-duration burst was found. The difference between the expected position of the $\gamma$-ray source and the $>100 \mathrm{MeV}$ emission centroid position computed by Ackermann et al. (2017) induces thinking about its possible causes.

The centroid position of the observed $\gamma$-ray emission can be due to superposition of a few different sources. For example, high-energy cosmic rays arriving from outside the solar system can produce cascades of secondary particles and $\gamma$ rays in the solar corona. Next, intense fluxes of energetic particles and emissions from the flare site can interact with dense streamers, producing secondaries, in particular $\gamma$-rays. Furthermore, the expanding CME is a low-density but huge 
target for both extra-solar cosmic rays and energetic flare emissions. Kahler and Ragot (2008) showed a possibility for high-energy $\gamma$-rays to be produced even in interactions between SEPs and solar wind. All of these presumable processes might influence the centroid position, while their effects are mostly expected in the lowest-energy part of the spectrum observed by Fermi/LAT.

Proceeding from these considerations, we attempted to find a possible dependence of the Fermi/LAT emission centroid position on the low-energy threshold. We analyzed the Level 1 Photon File available at fermi.gsfc.nasa.gov/ssc/data/ that included the SOL2014-09-01 event. The file presents the time, energy, and position (arrival direction) measured for each individual $\gamma$-ray photon out of numerous discrete sources detected during the observational interval recorded in the file. We calculated the emission centroid positions from these data, eliminating the $\gamma$-ray photons with energies below a given threshold $E_{\min }$. We did not reproduce the sophisticated method used by Ackermann et al. (2017) to reach the highest accuracy, making the calculations in the same way for each $E_{\min }$.

The centroid position that we found with $E_{\min }=100 \mathrm{MeV}$ was close to the result of Ackermann et al. (2017). Then we increased $E_{\min }$ in steps of $50 \mathrm{MeV}$ and found a monotonic displacement of the centroid position along the limb toward the southeast. The increase of $E_{\min }$ from $100 \mathrm{MeV}$ to $300 \mathrm{MeV}$ shifted it by $\approx 30^{\circ}$ toward the radio source GS2. Our experiment shows that the effects mentioned in this section can account for the discrepancy between the expected position of the source and the centroid position actually measured. This issue needs further study.

The energy dependence of the $\gamma$-ray centroid position is difficult to reconcile with the scenario proposed by Jin et al. (2018). We also recall the similarity of simulated HXR and $\gamma$-ray temporal profiles emitted from the trap after the same impulsive injection with those actually observed (Section 4.1.1). To fit within the scenario by Jin et al. (2018), accelerated electrons and protons of a common origin have to be separated and enter different structures to emit at the positions located far away from each other. Electrons have to come to the off-limb source GS2, while protons have to precipitate at the on-disk $\gamma$ ray centroid position. It seems difficult to realize this separation. GS2 appears to be a more probable source of both HXR and $\gamma$-ray emissions. According to Hudson (2017), the column density $n L$ required for the effective interaction of high-energy protons with ambient plasma can be accumulated in their numerous flights in a coronal trap (large $L$ ) and not necessarily be due to a large $n$ in the photosphere.

\subsection{Histories and Possible Roles of Shock Waves}

\subsubsection{Excitation and Evolution of Shock Waves}

As shown in our preceding case studies of shock-wave histories in a dozen events, the only initial shock-wave excitation scenario observed in flare-related eruptions is the impulsive-piston mechanism (Grechnev et al., 2018). Here, a sharply erupting flux rope produces strong MHD disturbance, whose initial speed is determined by the fast-mode speed $V_{\text {fast }}$, which is high in active regions 
$\left(V_{\text {fast }}>1000 \mathrm{~km} \mathrm{~s}^{-1}\right)$. Away from the eruption site, the $V_{\text {fast }}$ in the environment decreases both upwards and laterally, reaching $\approx 200 \mathrm{~km} \mathrm{~s}^{-1}$ above the quiet Sun. When a high-speed disturbance enters the lower- $V_{\text {fast }}$ environment, its profile steepens, and the disturbance rapidly becomes a shock wave. The shock formation is governed by the maximum acceleration of the eruption and the $V_{\text {fast }}$ falloff away from the eruption site, occurring presumably in $10-100$ seconds (Afanasyev, Uralov, and Grechnev, 2013). Then the shock wave propagates quasi-freely up to considerable distances from the Sun like a decelerating blast wave. Being highly efficient, the impulsive-piston scenario initially precedes the bow-shock excitation by the outer surface of a CME that is only possible when it becomes super-Alfvénic. The change to the bow-shock regime occurs later, if the trailing CME is fast (Grechnev et al., 2015, 2017).

The onset time of a shock wave excited in this way falls in the acceleration phase of the responsible eruption, i.e. during the rise phase of an HXR (microwave) burst. In a number of events, the acceleration of an eruption and shock onset time advanced the bursts by about two minutes (e.g. Grechnev et al., 2011a, 2013a, 2015, 2016, 2018). In several events, two shock waves excited within a few minutes by different eruptions were observed to follow each other. As shown in the articles listed, flare-generated shock waves are unlikely.

These conclusions shed light on the event in question. The presence of two EUV waves with onset times at 10:59:04 and 11:02:00 indicates excitation of two shock waves one after another by two presumable eruptions responsible for the HXR peaks observed by HEND around 11:02:20 and 11:04:30. Note that two bow shocks cannot be driven by a single CME. Most likely, two shock waves following each other eventually merge into a single, stronger shock propagating nearly radially (Grechnev et al., 2011a, 2013a, 2017). We do not have sufficient data about this process in our event and consider here a single shock wave relating it to the first one.

In the power-law description of a shock wave, the plasma density and wave speed become infinite in the origin $(t=0, x=0)$. This singularity is not important, because the shock forms at a certain distance from the origin, while the initial wave speed is determined by the fast-mode speed. From Figure $10 \mathrm{~b}$, the initial shock-wave speed in the radial direction was roughly about $3500 \mathrm{~km} \mathrm{~s}^{-1}$, which is a normal value for $V_{\text {fast }}$ in an active region. The kinematical histories of the shock waves at least up to $10 \mathrm{R}_{\odot}$ exhibit an overall quantitative agreement with the expected evolution outlined in the preceding paragraphs. Here we did not follow the shock-wave evolution in coronagraph images; the close correspondence of the calculated wave fronts to their signatures in the images and agreement with the measurements in the CME catalog was shown for several events previously (Grechnev et al., 2011a, b, 2014b, 2015, 2016, 2017, 2018).

There is no reason to presume the 1 September 2014 event to be exceptional. The shock-wave excitation and subsequent evolution appear to correspond to the impulsive-piston scenario outlined above. This shock-wave history turns out to be more complex than the bow-shock excitation by a super-Alfvénic piston alone, being, in fact, a combination of known scenarios (see, e.g., Vršnak and Cliver, 2008). Missing this circumstance can result in incorrect estimates and inadequate conclusions. 
In this respect, a question remains about the first estimate by Plotnikov, Rouillard, and Share (2017) of the shock-wave speed, which is an outlier in Figure 10. It follows from the description of the method in Rouillard et al. (2016) that the speed is calculated from the spatial separation of successive shock-front ellipsoids obtained in the three-dimensional reconstruction that appears to be justified. However, this method does not provide an estimate for the first and last speeds. Probably, this is the key to the problem, which seems to be systematic; the initial speeds also seem to be strongly underestimated for the three different events addressed in these articles. Another possible source of an additional error can be an apparent temporal difference between the first SDO/AIA images and a STEREO/EUVI or COR1 images presented in the articles; combination of the highest shock speed with the smallest size of its front can result in a large error for the initial point.

On the other hand, we emphasize the importance of a particular result of Rouillard et al. (2016) and Plotnikov, Rouillard, and Share (2017) about the ellipsoidal shape of the shock front that, in fact, confirms the scenario outlined above. A similar shape of the shock-wave front was predicted by Grechnev et al. (2011a), contrary to the bow shape with a Mach cone considered by Ontiveros and Vourlidas (2009). The reason is a three-dimensional expansion of the CME body ( $c f$. Vršnak and Cliver, 2008). Our expectations were later confirmed in studies by Kwon, Zhang, and Olmedo (2014) and Kwon, Zhang, and Vourlidas (2015). Elaboration of the shock-front reconstruction method by Rouillard et al. (2016) promises further progress in understanding coronal shock waves.

\subsubsection{Possible Particle Reacceleration by an Oblique Shock Wave}

As Hudson (2017) noted, our event resembles the SOL1969-03-30 event addressed by Frost and Dennis (1971), who considered the HXR emission observed in that event as evidence for two-stage electron acceleration (initially assumed by Wild, Smerd, and Weiss, 1963). The first-stage acceleration was associated with flare processes, while the shock front could be responsible for the second-stage acceleration. The two-stage acceleration can also apply to protons. Observations of the SOL2014-09-01 event might shed light on this issue.

An additional acceleration of high-energy protons in a static magnetic trap could be caused by a fast magnetosonic shock wave, whose front positions are shown in Figure 7 The shock front propagating with the phase velocity $V_{\text {sh }}$ intersects a part of the magnetic trap at an angle $[\psi]$ to the magnetic field $[\boldsymbol{B}]$. An important characteristic here is the velocity $[u]$ of the intersection point between a magnetic field line and the shock-front surface: $u=V_{\mathrm{sh}} \tan \psi$. We briefly discuss the case of an oblique shock wave with $u<c$.

High-energy particles with gyroradii considerably exceeding the shock-front thickness change their energy by virtue of the following effects. The first effect results from the first adiabatic-invariant $p_{\perp}^{2} / B$ conservation, where $p_{\perp}$ is a component of the particle momentum perpendicular to the magnetic field $p=m V /\left(1-V^{2} / c^{2}\right)^{1 / 2}, m$ is the rest mass of the particle, and $V$ is its velocity. Particles that are initially upstream of the shock, with pitch angles in the loss cone, pass into the downstream region of a stronger magnetic field and increase 
their transverse kinetic energy $K_{\perp}$. On the other hand, their longitudinal kinetic energy $K_{\|}$also changes because of the second effect of bouncing particles against the moving magnetic mirror of the shock front. The change $\Delta E=\Delta K$ in the total energy $E=m c^{2}+K_{\perp}+K_{\|}=m c^{2}+K$ depends on the initial pitch angle, velocity $u$, and $\psi$, and it can be either positive or negative.

The growth of the total energy due to the increasing $K_{\|}$is most conspicuous for particles reflected upstream after their interaction with the shock. In this case, $\Delta K=2 u p^{\prime} /\left(1-u^{2} / c^{2}\right)^{1 / 2}$ (Webb, Axford, and Terasawa, 1983), where $p^{\prime}$ is a gyrophase-averaged value of the particle's momentum projection on the shock-front surface in the frame moving along the front with the velocity $[u]$. In the non-relativistic limit $\left(u^{2} / c^{2} \ll 1\right), \Delta K=2 u\left(2 m K^{\prime}\right)^{1 / 2} /\left(1-u^{2} / c^{2}\right)^{1 / 2}$, where $K^{\prime}=\left(p^{\prime}\right)^{2} / 2 m$. The particles gain energy owing to the reflection from a moving magnetic mirror, i.e. the shock front for upstream particles with sufficiently large pitch angles. We are only interested in a qualitative analysis of the particle's behavior in a magnetic trap, through which a shock wave passes. Therefore, we replace the last relation with a rough proportion $\Delta K_{\|} \propto K_{\|}^{1 / 2}$. The higher the energy of a particle, the larger an increase in its energy and velocity per each reflection. The higher the particle velocity, the more reflections it has in bouncing between the moving shock front and the base of the magnetic trap. Thus, particle acceleration is accompanied by flattening of the initial energy spectrum.

After the shock-front passage, the magnetic-loop trap compresses and displaces following the wave. The magnetic field strength $B$ increases. Then the trap returns to its initial condition, and $B$ decreases to the initial value. With a decreasing $B$, the transversal energy $K_{\perp}$ of each particle decreases approximately as much as it increased in the interaction with the shock front because of the first adiabatic-invariant conservation. However, the longitudinal energy $K_{\|}$accumulated in the reflections from the shock front is retained, as well as the energy-spectrum flattening. This conclusion seems to correspond to the observations.

The completeness of this scheme for the proton acceleration in a trap is open to question. The acceleration mechanism based on reflections from the magnetic mirror in the shock front leads to an increase in the longitudinal energies of particles. This suggests a decrease in their pitch angles and possible precipitation into the loss cone of the magnetic trap. Precipitation of a fraction of energetic particles into the bases of the trap is expected to occur in the course of the oblique-shock propagation through a trap. This phenomenon might be manifested in the long-duration tail of the HXR emission observed by HEND in Figure 3 a. Precipitation may be reduced because of the electric field originating due to the charge separation in the front of a collisionless shock wave. Such electric field increases the transverse energy of reflected protons to prolong their confinement in the trap.

\section{Summary and Conclusions}

A combined analysis of observations of the far-side SOL2014-09-01 event from different vantage points has revealed the following circumstances. 
i The lift-off of a hot (about $10 \mathrm{MK}$ ) blob has been detected, which probably was an erupting flux rope. The blob rose radially and became the CME core.

ii Unocculted flare emission consisted of two HXR peaks with similar spectra separated by 2.5 minutes.

iii Each of the two flare peaks was preceded by the appearance of a shock wave by two to three minutes.

iv The first HXR peak was manifested in a static off-limb gyrosynchrotron radio source of a corresponding duration and spectrum.

v The second HXR peak gave rise to a different static off-limb gyrosynchrotron radio source of a considerably longer duration and harder spectrum. This radio source was located in a system of long loops.

vi The long-duration gyrosynchrotron burst from the second source was almost identical in shape with the HXR burst observed from the Earth's direction and rather similar to the $>100 \mathrm{MeV} \gamma$-ray burst. All of these emissions could be produced by populations of electrons and protons injected into the long loops during the second flare burst and trapped there for a long time.

vii The harder spectrum of the long-duration burst relative to the injection could be due to reacceleration of the particles trapped in closed loops by the second shock wave.

viii The observations indicate that the sources of the gyrosynchrotron, HXR, and $\gamma$-ray emissions had a common location. It was considerably displaced with respect to the $>100 \mathrm{MeV} \gamma$-ray emission centroid position. A probable key to the discrepancy is a contribution of $\gamma$-rays coming from high coronal structures and possibly the CME. The role of non-solar high-energy cosmic rays is not excluded.

These findings can be reconciled within the following scenario. Two sharp eruptions probably occurred in Active Region 12158 with an interval of about 2.5 minutes. Each eruption impulsively excited a blast-wave-like shock, on the one hand, and resulted in strong particle acceleration in the flare site, on the other hand. Manifestations of the first flare peak were observed from the Earth's direction as an impulsive brightening of the arcade top. During the second peak, accelerated electrons and protons were injected into long loops, where they become trapped for a long time. The second shock wave possibly hit these loops obliquely, which resulted in reacceleration of trapped flare-accelerated electrons and protons. This presumable episode was not crucial; the long-duration gyrosynchrotron, hard X-ray, and $\gamma$-ray emissions were radiated from trapped particles, while reacceleration hardened their spectra. A presumable scenario with a shock-acceleration of particles high in the corona and their return to the solar surface along open magnetic structures meets basic difficulties and is not confirmed by observations. 
The region of trapped electrons and protons was located above the limb. Its connection to the Earth-facing solar surface near the Equator is not excluded, but does not seem to be necessary.

While our analysis sheds additional light on this event, a number of issues remain to be addressed. We hope that our results will highlight possible ways for future studies.

Acknowledgments This work is dedicated to the memory of M.A. Livshits, who initiated this study. We appreciate discussions with E. Carley, N. Vilmer, and H. Hudson, and useful remarks of the anonymous reviewer. We thank the NASA/SDO and the AIA and HMI science teams; the NASA's STEREO/SECCHI science and instrument teams; the teams of the SWAP telescope on the ESA's PROBA 2 spacecraft, the NASA's Fermi Gamma-Ray Space Telescope, the Wind/Konus team at the Ioffe Institute, the USAF RSTN network, and LASCO on $\mathrm{SOHO}$. SOHO is a project of international cooperation between ESA and NASA. We thank the team maintaining the CME Catalog at the CDAW Data Center by NASA and the Catholic University of America in cooperation with the Naval Research Laboratory.

The studies presented in Sections 12.1 and 2.2 were carried out by V. Kiselev and I. Grigorieva and funded by the Russian Foundation of Basic Research under grant 17-3250040_mol_nr. V. Grechnev, A. Kochanov, and A. Uralov (Sections 3 4 and 5) were funded by the Russian Science Foundation under grant 18-12-00172.

\section{Disclosure of Potential Conflicts of Interest}

The authors claim that they have no conflicts of interest.

\section{References}

Ackermann, M., Allafort, A., Baldini, L., Barbiellini, G., Bastieri, D., Bellazzini, R., Bissaldi, E., Bonino, R., Bottacini, E., Bregeon, J., et al.: 2017, Fermi-LAT Observations of Highenergy Behind-the-limb Solar Flares. Astrophys. J. 835, 219. DOI ADS

Afanasyev, A.N., Uralov, A.M., Grechnev, V.V.: 2013, Propagation of a fast magnetoacoustic shock wave in the magnetosphere of an active region. Astronomy Reports 57, 594. DOI ADS

Akimov, V.V., Ambrož, P., Belov, A.V., Berlicki, A., Chertok, I.M., Karlický, M., Kurt, V.G., Leikov, N.G., Litvinenko, Y.E., Magun, A., Minko-Wasiluk, A., Rompolt, B., Somov, B.V.: 1996, Evidence for prolonged acceleration based on a detailed analysis of the long-duration solar gamma-ray flare of June 15, 1991. Solar Phys. 166, 107. DOI ADS

Aptekar, R.L., Frederiks, D.D., Golenetskii, S.V., Ilynskii, V.N., Mazets, E.P., Panov, V.N., Sokolova, Z.J., Terekhov, M.M., Sheshin, L.O., Cline, T.L., Stilwell, D.E.: 1995, Konus-W Gamma-Ray Burst Experiment for the GGS Wind Spacecraft. Space Sci. Rev. 71, 265. DOI. ADS

Aschwanden, M.J.: 2004a, Physics of the Solar Corona. An Introduction, Praxis Publishing Ltd., Chichester, UK. Chap. 12. ADS

Aschwanden, M.J.: 2004b, Pulsed Particle Injection in a Reconnection-Driven Dynamic Trap Model in Solar Flares. Astrophys. J. 608, 554. DOI ADS

Aschwanden, M.J.: 2012, GeV Particle Acceleration in Solar Flares and Ground Level Enhancement (GLE) Events. Space Sci. Rev. 171, 3. DOI ADS

Atwood, W.B., Abdo, A.A., Ackermann, M., Althouse, W., Anderson, B., Axelsson, M., Baldini, L., Ballet, J., Band, D.L., Barbiellini, G., et al.: 2009, The Large Area Telescope on the Fermi Gamma-Ray Space Telescope Mission. Astrophys. J. 697, 1071. DOI ADS

Aurass, H.: 2003, Solar Radio Bursts after Yohkoh and SOHO. Hvar Observatory Bulletin 27, 103. ADS

Aurass, H., Vršnak, B., Mann, G.: 2002, Shock-excited radio burst from reconnection outflow jet? Astron. Astrophys. 384, 273. DOI ADS 
Bemporad, A., Soenen, A., Jacobs, C., Landini, F., Poedts, S.: 2010, Side Magnetic Reconnections Induced by Coronal Mass Ejections: Observations and Simulations. Astrophys. J. 718, 251. DOI ADS

Berghmans, D., Hochedez, J.F., Defise, J.M., Lecat, J.H., Nicula, B., Slemzin, V., Lawrence, G., Katsyiannis, A.C., van der Linden, R., Zhukov, A., et al.: 2006, SWAP onboard PROBA 2, a new EUV imager for solar monitoring. Adv. Space Res. 38, 1807. DOI ADS.

Boynton, W.V., Feldman, W.C., Mitrofanov, I.G., Evans, L.G., Reedy, R.C., Squyres, S.W., Starr, R., Trombka, J.I., D'Uston, C., Arnold, J.R., et al.: 2004, The Mars Odyssey GammaRay Spectrometer Instrument Suite. Space Sci. Rev. 110, 37. DOI ADS

Brueckner, G.E., Howard, R.A., Koomen, M.J., Korendyke, C.M., Michels, D.J., Moses, J.D., Socker, D.G., Dere, K.P., Lamy, P.L., Llebaria, A., et al.: 1995, The Large Angle Spectroscopic Coronagraph (LASCO). Solar Phys. 162, 357. DOI ADS

Carley, E.P., Vilmer, N., Simões, P.J.A., Ó Fearraigh, B.: 2017, Estimation of a coronal mass ejection magnetic field strength using radio observations of gyrosynchrotron radiation. Astron. Astrophys. 608, A137. DOI ADS

Cheng, X., Zhang, J., Liu, Y., Ding, M.D.: 2011, Observing Flux Rope Formation During the Impulsive Phase of a Solar Eruption. Astrophys. J. Lett. 732, L25. DOI ADS

Chertok, I.M., Belov, A.V., Grechnev, V.V.: 2015, A Simple Way to Estimate the Soft X-ray Class of Far-Side Solar Flares Observed with STEREO/EUVI. Solar Phys. 290, 1947. DOI ADS

Chupp, E.L., Ryan, J.M.: 2009, High energy neutron and pion-decay gamma-ray emissions from solar flares. Research in Astron. Astrophys. 9, 11. DOI ADS

Cliver, E.W., Kahler, S.W., Vestrand, W.T.: 1993, On the Origin of Gamma-Ray Emission from the Behind-the-Limb Flare on 29 September 1989. In: Leahy, D.A., Hicks, R.B., Venkatesan, D. (eds.) Singapore: World Scientific, Internat. Cosmic Ray Conf. 3, 91. ADS

Dulk, G.A.: 1985, Radio emission from the sun and stars. Annu. Rev. Astron. Astrophys. 23, 169. DOI ADS

Dulk, G.A., Marsh, K.A.: 1982, Simplified expressions for the gyrosynchrotron radiation from mildly relativistic, nonthermal and thermal electrons. Astrophys. J. 259, 350. DOI ADS

Forrest, D.J., Vestrand, W.T., Chupp, E.L., Rieger, E., Cooper, J.F., Share, G.H.: 1985, Neutral pion production in solar flares. International Cosmic Ray Conference 4, 146. ADS

Frost, K.J., Dennis, B.R.: 1971, Evidence from Hard X-Rays for Two-Stage Particle Acceleration in a Solar Flare. Astrophys. J. 165, 655. DOI ADS.

Gary, G.A., Moore, R.L.: 2004, Eruption of a Multiple-Turn Helical Magnetic Flux Tube in a Large Flare: Evidence for External and Internal Reconnection That Fits the Breakout Model of Solar Magnetic Eruptions. Astrophys. J. 611, 545. DOI. ADS

Grechnev, V.V., Uralov, A.M., Slemzin, V.A., Chertok, I.M., Kuzmenko, I.V., Shibasaki, K.: 2008a, Absorption Phenomena and a Probable Blast Wave in the 13 July 2004 Eruptive Event. Solar Phys. 253, 263. DOI ADS

Grechnev, V.V., Kurt, V.G., Chertok, I.M., Uralov, A.M., Nakajima, H., Altyntsev, A.T., Belov, A.V., Yushkov, B.Y., Kuznetsov, S.N., Kashapova, L.K., Meshalkina, N.S., Prestage, N.P.: 2008b, An Extreme Solar Event of 20 January 2005: Properties of the Flare and the Origin of Energetic Particles. Solar Phys. 252, 149. DOI ADS

Grechnev, V.V., Uralov, A.M., Chertok, I.M., Kuzmenko, I.V., Afanasyev, A.N., Meshalkina, N.S., Kalashnikov, S.S., Kubo, Y.: 2011a, Coronal Shock Waves, EUV Waves, and Their Relation to CMEs. I. Reconciliation of "EIT Waves", Type II Radio Bursts, and Leading Edges of CMEs. Solar Phys. 273, 433. DOI ADS

Grechnev, V.V., Afanasyev, A.N., Uralov, A.M., Chertok, I.M., Eselevich, M.V., Eselevich, V.G., Rudenko, G.V., Kubo, Y.: 2011b, Coronal Shock Waves, EUV Waves, and Their Relation to CMEs. III. Shock-Associated CME/EUV Wave in an Event with a Two-Component EUV Transient. Solar Phys. 273, 461. DOI ADS.

Grechnev, V.V., Kuzmenko, I.V., Chertok, I.M., Uralov, A.M.: 2011c, Solar flare-related eruptions followed by long-lasting occultation of the emission in the He II $304 \AA$ line and in microwaves. Astron. Rep. 55, 637. DOI ADS

Grechnev, V.V., Kiselev, V.I., Uralov, A.M., Meshalkina, N.S., Kochanov, A.A.: 2013a, An Updated View of Solar Eruptive Flares and the Development of Shocks and CMEs: History of the 2006 December 13 GLE-Productive Extreme Event. Publ. Astron. Soc. Japan 65, S9. DOI ADS.

Grechnev, V.V., Kuz'menko, I.V., Uralov, A.M., Chertok, I.M., Kochanov, A.A.: 2013b, Microwave Negative Bursts as Indications of Reconnection Between Eruptive Filaments and a Large-Scale Coronal Magnetic Environment. Publ. Astron. Soc. Japan 65, S10. DOI ADS 
Grechnev, V.V., Uralov, A.M., Slemzin, V.A., Chertok, I.M., Filippov, B.P., Rudenko, G.V., Temmer, M.: 2014a, A Challenging Solar Eruptive Event of 18 November 2003 and the Causes of the 20 November Geomagnetic Superstorm. I. Unusual History of an Eruptive Filament. Solar Phys. 289, 289. DOI ADS

Grechnev, V.V., Uralov, A.M., Chertok, I.M., Slemzin, V.A., Filippov, B.P., Egorov, Y.I., Fainshtein, V.G., Afanasyev, A.N., Prestage, N.P., Temmer, M.: 2014b, A Challenging Solar Eruptive Event of 18 November 2003 and the Causes of the 20 November Geomagnetic Superstorm. II. CMEs, Shock Waves, and Drifting Radio Bursts. Solar Phys. 289, 1279. DOI. ADS

Grechnev, V.V., Uralov, A.M., Kuzmenko, I.V., Kochanov, A.A., Chertok, I.M., Kalashnikov, S.S.: 2015, Responsibility of a Filament Eruption for the Initiation of a Flare, CME, and Blast Wave, and its Possible Transformation into a Bow Shock. Solar Phys. 290, 129. DOI] ADS

Grechnev, V.V., Uralov, A.M., Kochanov, A.A., Kuzmenko, I.V., Prosovetsky, D.V., Egorov, Y.I., Fainshtein, V.G., Kashapova, L.K.: 2016, A Tiny Eruptive Filament as a Flux-Rope Progenitor and Driver of a Large-Scale CME and Wave. Solar Phys. 291, 1173. DOI ADS

Grechnev, V.V., Kiselev, V.I., Uralov, A.M., Klein, K.-L., Kochanov, A.A.: 2017, The 26 December 2001 Solar Eruptive Event Responsible for GLE63: III. CME, Shock Waves, and Energetic Particles. Solar Phys. 292, 102. DOI ADS

Grechnev, V.V., Lesovoi, S.V., Kochanov, A.A., Uralov, A.M., Altyntsev, A.T., Gubin, A.V., Zhdanov, D.A., Ivanov, E.F., Smolkov, G.Y., Kashapova, L.K.: 2018, Multi-instrument view on solar eruptive events observed with the Siberian Radioheliograph: From detection of small jets up to development of a shock wave and CME. J. Atmos. Solar-Terr. Phys. 174, 46. DOI ADS

Guidice, D.A.: 1979, Sagamore Hill Radio Observatory, Air Force Geophysics Laboratory, Hanscom Air Force Base, Massachusetts 01731. Report. Bull. Am. Astron. Soc. 11, 311. ADS

Guidice, D.A., Cliver, E.W., Barron, W.R., Kahler, S.: 1981, The Air Force RSTN System. Bull. Am. Astron. Soc. 13, 553. ADS

Howard, R.A., Moses, J.D., Vourlidas, A., Newmark, J.S., Socker, D.G., Plunkett, S.P., Korendyke, C.M., Cook, J.W., Hurley, A., Davila, J.M., et al.: 2008, Sun Earth Connection Coronal and Heliospheric Investigation (SECCHI). Space Sci. Rev. 136, 67. DOI ADS

Hudson, H., Ryan, J.: 1995, High-Energy Particles In Solar Flares. Annu. Rev. Astron. Astrophys. 33, 239. DOI ADS

Hudson, H.S.: 2017, The Relationship between Long-Duration Gamma-Ray Flares and Solar Cosmic Rays. In: Foullon, C., Malandraki, O.E. (eds.) Space Weather of the Heliosphere: Processes and Forecasts, IAU Symp. 335, 49. DOI. ADS

Jin, M., Petrosian, V., Liu, W., Nitta, N.V., Omodei, N., Rubio da Costa, F., Effenberger, F., Li, G., Pesce-Rollins, M., Allafort, A., Manchester, W. IV: 2018, Data-driven Simulations of Magnetic Connectivity in Behind-the-Limb $\gamma$-ray Flares and Associated Coronal Mass Ejections. ArXiv e-prints, submitted to Astrophys. J. ADS

Kahler, S.W.: 2001, Origin and properties of solar energetic particles in space. In: Song, P., Singer, H.J., Siscoe, G.L. (eds.) Space Weather, Am. Geophys. Un. Geophys. Mono. Ser. 125, 109. DOI ADS

Kahler, S.W., Ragot, B.R.: 2008, Remote Sensing of Gamma-Ray Emission from Solar Energetic Proton Interactions with the Solar Wind. Astrophys. J. 675, 846. DOI ADS

Kaiser, M.L., Kucera, T.A., Davila, J.M., St. Cyr, O.C., Guhathakurta, M., Christian, E.: 2008, The STEREO Mission: An Introduction. Space Sci. Rev. 136, 5. DOI ADS

Kallenrode, M.-B.: 2003, Current views on impulsive and gradual solar energetic particle events J. Phys. G Nucl. Phys, 29, 965. ADS

Kerdraon, A., Delouis, J.-M.: 1997, The Nançay Radioheliograph. In: Trottet, G. (ed.) Coronal Physics from Radio and Space Observations, Lecture Notes in Physics 483. Springer, Berlin, 192. DOI ADS

Kiplinger, A.L.: 1995, Comparative Studies of Hard X-Ray Spectral Evolution in Solar Flares with High-Energy Proton Events Observed at Earth. Astrophys. J. 453, 973. DOI ADS

Kochanov, A.A., Anfinogentov, S.A., Prosovetsky, D.V., Rudenko, G.V., Grechnev, V.V.: 2013, Imaging of the Solar Atmosphere by the Siberian Solar Radio Telescope at $5.7 \mathrm{GHz}$ with an Enhanced Dynamic Range. Publ. Astron. Soc. Japan 65, S19. DOI ADS

Kundu, M.R., White, S.M., Shibasaki, K., Sakurai, T., Grechnev, V.V.: 2001, Spatial Structure of Simple Spiky Bursts at Microwave/Millimeter Wavelengths. Astrophys. J. 547, 1090. DOI. ADS 
Kurt, V.G., Yushkov, B.Y., Kudela, K., Galkin, V.I.: 2010, High-energy gamma radiation of solar flares as an indicator of acceleration of energetic protons. Cos. Res. 48, 70. DOI ADS

Kuzmenko, I.V., Grechnev, V.V.: 2017, Development and Parameters of a Non-Self-Similar CME Caused by the Eruption of a Quiescent Prominence. Solar Phys. 292, 143. DOI

Kuznetsov, A.A., Nita, G.M., Fleishman, G.D.: 2011, Three-dimensional Simulations of Gyrosynchrotron Emission from Mildly Anisotropic Nonuniform Electron Distributions in Symmetric Magnetic Loops. Astrophys. J. 742, 87. DOI. ADS

Kuznetsov, S.N., Kurt, V.G., Yushkov, B.Y., Kudela, K., Galkin, V.I.: 2011, Gamma-Ray and High-Energy-Neutron Measurements on CORONAS-F during the Solar Flare of 28 October 2003. Solar Phys. 268, 175. DOI ADS

Kwon, R.-Y., Zhang, J., Olmedo, O.: 2014, New Insights into the Physical Nature of Coronal Mass Ejections and Associated Shock Waves within the Framework of the Three-dimensional Structure. Astrophys. J. 794, 148. DOI ADS.

Kwon, R.-Y., Zhang, J., Vourlidas, A.: 2015, Are Halo-like Solar Coronal Mass Ejections Merely a Matter of Geometric Projection Effects? Astrophys. J. Lett. 799, L29. DOI ADS

Lecacheux, A.: 2000, The Nançay Decameter Array: A Useful Step Towards Giant, New Generation Radio Telescopes for Long Wavelength Radio Astronomy. In: Stone, R.G., Weiler, K.W., Goldstein, M.L., Bougerot, J.-L. (eds.) Radio Astronomy at Long Wavelengths, tutorials and reviews from an AGU Chapman conference, Am. Geophys. Un. Geophys. Mono. Ser. 119, 321. DOI ADS

Lemen, J.R., Title, A.M., Akin, D.J., Boerner, P.F., Chou, C., Drake, J.F., Duncan, D.W., Edwards, C.G., Friedlaender, F.M., Heyman, G.F., et al.: 2012, The Atmospheric Imaging Assembly (AIA) on the Solar Dynamics Observatory (SDO). Solar Phys. 275, 17. DOI ADS

Livshits, M.A., Belov, A.V.: 2004, When and Where are Solar Cosmic Rays Accelerated Most Efficiently? Astron. Rep. 48, 665. DOI ADS

Livshits, M.A., Zimovets, I.V., Golovin, D.V., Nizamov, B.A., Vybornov, V.I., Mitrofanov, I.G., Kozyrev, A.S., Litvak, M.L., Sanin, A.B., Tretyakov, V.I.: 2017, Catalog of hard Xray solar flares detected with Mars Odyssey/HEND from the Mars orbit in 2001-2016. Astron. Rep. 61, 791. DOI ADS

Mandzhavidze, N., Ramaty, R.: 1992, Gamma rays from pion decay - Evidence for long-term trapping of particles in solar flares. Astrophys. J. Lett. 396, L111. DOI ADS

Masson, S., Antiochos, S.K., DeVore, C.R.: 2013, A Model for the Escape of Solar-flareaccelerated Particles. Astrophys. J. 771, 82. DOI. ADS.

Meegan, C., Lichti, G., Bhat, P.N., Bissaldi, E., Briggs, M.S., Connaughton, V., Diehl, R., Fishman, G., Greiner, J., Hoover, A.S., et al.: 2009, The Fermi Gamma-ray Burst Monitor. Astrophys. J. 702, 791. DOI ADS

Melnikov, V.F., Magun, A.: 1998, Spectral Flattening During Solar Radio Bursts At Cm-mm Wavelengths and the Dynamics of Energetic Electrons in a Flare Loop. Solar Phys. 178, 153. DOI ADS

Melrose, D.B., Brown, J.C.: 1976, Precipitation in trap models for solar hard X-ray bursts. Mon. Not. Roy. Astron. Soc. 176, 15. DOI ADS

Meshalkina, N.S., Uralov, A.M., Grechnev, V.V., Altyntsev, A.T., Kashapova, L.K.: 2009, Eruptions of Magnetic Ropes in Two Homologous Solar Events of 2002 June 1 and 2: a Key to Understanding an Enigmatic Flare. Publ. Astron. Soc. Japan 61, 791. DOI ADS

Metcalf, T.R., Alexander, D.: 1999, Coronal Trapping of Energetic Flare Particles:Yohkoh/HXT Observations. Astrophys. J. 522, 1108. DOI ADS

Nitta, N.V., Aschwanden, M.J., Boerner, P.F., Freeland, S.L., Lemen, J.R., Wuelser, J.-P.: 2013, Soft X-ray Fluxes of Major Flares Far Behind the Limb as Estimated Using STEREO EUV Images. Solar Phys. 288, 241. DOI ADS

Ontiveros, V., Vourlidas, A.: 2009, Quantitative Measurements of Coronal Mass EjectionDriven Shocks from LASCO Observations. Astrophys. J. 693, 267. DOI ADS

Patsourakos, S., Vourlidas, A., Stenborg, G.: 2013, Direct Evidence for a Fast Coronal Mass Ejection Driven by the Prior Formation and Subsequent Destabilization of a Magnetic Flux Rope. Astrophys. J. 764, 125. DOI ADS

Pesce-Rollins, M., Omodei, N., Petrosian, V., Liu, W., Rubio da Costa, F., Allafort, A., Fermi-LAT Collaboration: 2015, Fermi Large Area Telescope observations of high-energy gamma-ray emission from behind-the-limb solar flares. In: Borisov, A.S., Denisova, V.G., Guseva, Z.M., Kanevskaya, E.A., Kogan, M.G., Morozov, A.E., Puchkov, V.S., Pyatovsky, S.E., Shoziyoev, G.P., Smirnova, M.D., Vargasov, A.V., Galkin, V.I., Nazarov, S.I., 
Mukhamedshin, R.A. (eds.) Proc. 34th Internat. Cosmic Ray Conf. (ICRC2015) 34, Hague, Netherlands, 128. ADS

Plotnikov, I., Rouillard, A.P., Share, G.H.: 2017, The magnetic connectivity of coronal shocks from behind-the-limb flares to the visible solar surface during $\gamma$-ray events. Astron. Astrophys. 608, A43. DOI ADS

Ramaty, R., Mandzhavidze, N.: 2000, Gamma-rays from Solar Flares. In: Martens, P.C.H., Tsuruta, S., Weber, M.A. (eds.) Highly Energetic Physical Processes and Mechanisms for Emission from Astrophysical Plasmas, IAU Symp. 195, 123. ADS

Ramaty, R., Kozlovsky, B., Lingenfelter, R.E.: 1975, Solar gamma rays. Space Sci. Rev. 18, 341. DOI ADS

Reames, D.V.: 2013, The Two Sources of Solar Energetic Particles. Space Sci. Rev. 175, 53. DOI ADS

Rouillard, A.P., Plotnikov, I., Pinto, R.F., Tirole, M., Lavarra, M., Zucca, P., Vainio, R., Tylka, A.J., Vourlidas, A., De Rosa, M.L., et al.: 2016, Deriving the Properties of Coronal Pressure Fronts in 3D: Application to the 2012 May 17 Ground Level Enhancement. Astrophys. J. 833, 45. DOI ADS

Ryan, J.M.: 2000, Long-Duration Solar Gamma-Ray Flares. Space Sci. Rev. 93, 581. ADS

Saito, K., Makita, M., Nishi, K., Hata, S.: 1970, A non-spherical axisymmetric model of the solar K corona of the minimum type. Ann. Tokyo Astron. Observ. 12, 53. ADS.

Scherrer, P.H., Schou, J., Bush, R.I., Kosovichev, A.G., Bogart, R.S., Hoeksema, J.T., Liu, Y., Duvall, T.L., Zhao, J., Title, A.M., et al.: 2012, The Helioseismic and Magnetic Imager (HMI) Investigation for the Solar Dynamics Observatory (SDO). Solar Phys. 275, 207. DOI. ADS

Share, G.H., Murphy, R.J., Tolbert, A.K., Dennis, B.R., White, S.M., Schwartz, R.A., Tylka, A.J.: 2017, Characteristics of Sustained $>100 \mathrm{MeV}$ Gamma-ray Emission Associated with Solar Flares. ArXiv e-print 1711.01511, submitted to Astrophys. J. Supp. ADS

Silva, A.V.R., Wang, H., Gary, D.E.: 2000, Correlation of Microwave and Hard X-Ray Spectral Parameters. Astrophys. J. 545, 1116. DOI ADS

Uralov, A.M., Grechnev, V.V., Rudenko, G.V., Myshyakov, I.I., Chertok, I.M., Filippov, B.P., Slemzin, V.A.: 2014, A Challenging Solar Eruptive Event of 18 November 2003 and the Causes of the 20 November Geomagnetic Superstorm. III. Catastrophe of the Eruptive Filament at a Magnetic Null Point and Formation of an Opposite-Handedness CME. Solar Phys. 289, 3747. DOI ADS

van Driel-Gesztelyi, L., Baker, D., Török, T., Pariat, E., Green, L.M., Williams, D.R., Carlyle, J., Valori, G., Démoulin, P., Kliem, B., Long, D.M., Matthews, S.A., Malherbe, J.-M.: 2014, Coronal Magnetic Reconnection Driven by CME Expansion-the 2011 June 7 Event. Astrophys. J. 788, 85. DOI ADS

Vilmer, N., MacKinnon, A.L., Hurford, G.J.: 2011, Properties of Energetic Ions in the Solar Atmosphere from $\gamma$-Ray and Neutron Observations. Space Sci. Rev. 159, 167. DOI ADS

Vršnak, B., Cliver, E.W.: 2008, Origin of Coronal Shock Waves. Invited Review. Solar Phys. 253, 215. DOI ADS

Webb, G.M., Axford, W.I., Terasawa, T.: 1983, On the drift mechanism for energetic charged particles at shocks. Astrophys. J. 270, 537. DOI ADS

White, S.M., Krucker, S., Shibasaki, K., Yokoyama, T., Shimojo, M., Kundu, M.R.: 2003, Radio and Hard X-Ray Images of High-Energy Electrons in an X-Class Solar Flare. Astrophys. J. Lett. 595, L111. DOI. ADS.

White, S.M., Benz, A.O., Christe, S., Fárník, F., Kundu, M.R., Mann, G., Ning, Z., Raulin, J.P., Silva-Válio, A.V.R., Saint-Hilaire, P., Vilmer, N., Warmuth, A.: 2011, The Relationship Between Solar Radio and Hard X-ray Emission. Space Sci. Rev. 159, 225. DOI. ADS.

Wild, J.P., Smerd, S.F., Weiss, A.A.: 1963, Solar Bursts. Annu. Rev. Astron. Astrophys. 1, 291. DOI ADS

Yashiro, S., Gopalswamy, N., Michalek, G., St. Cyr, O.C., Plunkett, S.P., Rich, N.B., Howard, R.A.: 2004, A catalog of white light coronal mass ejections observed by the SOHO spacecraft. J. Geophys. Res.(Space Physics) 109, A07105. DOI ADS

Zimovets, I., Vilmer, N., Chian, A.C.-L., Sharykin, I., Struminsky, A.: 2012, Spatially resolved observations of a split-band coronal type II radio burst. Astron. Astrophys. 547, A6. DOI ADS 